\title{
FOURIER TRANSFORMS RELATED TO A ROOT SYSTEM OF RANK 1
}

\section{WOLTER GROENEVELT*}

\author{
Korteweg-De Vries \\ Instituut voor Wiskunde \\ Universiteit van Amsterdam \\ Plantage Muidergracht 24 \\ 1018 TV Amsterdam, The Netherlands \\ wgroenev@science.uva.nl
}

\begin{abstract}
We introduce an algebra $\mathcal{H}$ consisting of difference-reflection operators and multiplication operators that can be considered as a $q=1$ analogue of Sahi's double affine Hecke algebra related to the affine root system of type $\left(C_{1}^{\vee}, C_{1}\right)$. We study eigenfunctions of a DunklCherednik-type operator in the algebra $\mathcal{H}$, and the corresponding Fourier transforms. These eigenfunctions are nonsymmetric versions of the Wilson polynomials and the Wilson functions.
\end{abstract}

\section{Introduction}

Cherednik's double affine Hecke algebras and their degenerate versions are very useful for studying Macdonald orthogonal polynomials and generalized Fourier transforms, see, e.g., Cherednik [2], [3], Macdonald [15], Opdam [19], Sahi [20], Stokman [23]. In rank 1 this approach leads to new interpretations and new results for many well-known orthogonal polynomials of (basic) hypergeometric type and corresponding integral transforms. In this paper we are interested in generalized Fourier transforms related to the rank 1 root system of type $\left(C^{\vee}, C\right)$. The kernels in our Fourier transforms are nonsymmetric versions of the Wilson polynomials [24] and the Wilson functions [8].

Sahi [20] defined a double affine Hecke algebra $\mathcal{H}_{q}$ related to the nonreduced root system of type $\left(C^{\vee}, C\right)$, using Noumi's representation of the affine Hecke algebra associated to root systems of type $C$ [17]. Sahi's algebra in rank 1 is very useful for studying Askey-Wilson polynomials [1] and Askey-Wilson functions [11], see Noumi and Stokman [18], Stokman [23], and Sahi [21]. In fact, Sahi's double affine Hecke algebra $\mathcal{H}_{q}$ is used in [20], [21], [22] to study the Koornwinder-Macdonald polynomials, the multivariable versions of the Askey-Wilson polynomials introduced by Koornwinder [13]. In rank 1 the double affine Hecke algebra $\mathcal{H}_{q}$ depends, besides $q$, on four independent parameters, corresponding to the number of $\mathcal{W}$-orbits in the root system, where $\mathcal{W}$ is the affine Weyl group. These parameters correspond to the four parameters of the

\footnotetext{
DOI: $10.1007 / \mathrm{s} 00031-005-1124-5$.

${ }^{*}$ The author is supported by the Netherlands Organization for Scientific Research (NWO) for the Vidi-project "Symmetry and modularity in exactly solvable models." Parts of this research were done during the author's stay at the Department of Mathematics at Chalmers University of Technology and the Göteborg University in Sweden, supported by an NWOTALENT stipendium of the Netherlands Organization for Scientific Research (NWO).

Received November 9, 2005. Accepted April 3, 2006. Published online February 22, 2007.
} 
Askey-Wilson polynomials. A certain isomorphism of the double affine Hecke algebra leads to the duality property for the Askey-Wilson polynomials and functions. This property basically says that the geometric and spectral variable can be interchanged. In the limit $q \rightarrow 1$ the double affine Hecke algebra goes over into the degenerate double affine Hecke algebra, see [4] for these limits in the case of reduced root systems. This limit transition between algebras corresponds to the limit transition between AskeyWilson polynomials and Jacobi polynomials. However, the polynomials and the algebra obtained in the limit depend only on two independent parameters instead of four, and there is no longer a duality property. For $q \rightarrow 1$ the Askey-Wilson polynomials also have a limit that depends on all four parameters, namely, the Wilson polynomials. It is a natural question to ask if there is a degenerate double affine Hecke algebra related to the Wilson polynomials.

In this paper we construct such an algebra related to the Wilson polynomials. We define a representation of the affine Weyl group which resembles Noumi's representation of the affine Hecke algebra associated to root systems of type $C$. For this representation we use difference operators, similar to the difference operators used by Cherednik [4] to obtain the inverse of the Harish-Chandra transform. With our representation of the affine Weyl group, we construct an algebra $\mathcal{H}$ which is a new example of a degenerate Hecke algebra. It is shown that $\mathcal{H}$ is a degeneration of Sahi's double affine Hecke algebra of type $\left(C^{\vee}, C\right)$ in rank 1. In particular, $\mathcal{H}$ depends on four independent parameters. We remark that there exists a degeneration of Sahi's algebra in rank $n$ similar to the algebra $\mathcal{H}$ studied in this paper. In the limit the $C_{n}$ braid relations are deformed, so in rank $n$ the algebra $\mathcal{H}$ is not obtained from the representation of the Weyl group for the root system of type $\left(C_{n}^{\vee}, C_{n}\right)$. The rank $n$ case will be studied in a future paper.

As in Sahi's algebra, we have a duality isomorphism $\sigma$ for the algebra $\mathcal{H}$. To this isomorphism $\sigma$ one can associate a Fourier transform $\mathbb{F}: V \rightarrow W$, which is a linear map that intertwines the actions of $\mathcal{H}$ and $\sigma(\mathcal{H})$ on the vector spaces $V$ and $W$, respectively, that is, $\mathbb{F} \circ X=\sigma(X) \circ \mathbb{F}$ for all $X \in \mathcal{H}$. We consider Fourier transforms that can be written as integral transforms with kernels the eigenfunctions of the Dunkl-Cherednik-type operator. The eigenfunctions of a Dunkl-Cherednik-type operator in our representation are nonsymmetric versions of the Wilson polynomials and the Wilson functions.

We study the nonsymmetric Wilson polynomials using the degenerate affine Hecke algebra $\mathcal{H}$, which is very similar to the study of nonsymmetric Askey-Wilson polynomials in [18]. The nonsymmetric Wilson functions, however, cannot be treated in the same way as the nonsymmetric Askey-Wilson functions (the rank 1 Cherednik kernel in [23]). For instance, the nonsymmetric Askey-Wilson functions are defined in [23] as a sum of nonsymmetric Askey-Wilson polynomials, but a similar sum of nonsymmetric Wilson polynomials does not converge absolutely.

Recently, Zhang [25] gave an interpretion of the (symmetric) multivariable Wilson polynomials in the representation theory of the degenerate double affine Hecke algebra by showing that the multivariable Wilson polynomials can be obtained from symmetric multivariable Jacobi polynomials using the Harish-Chandra transform. For rank 1 this was proved by Koornwinder [12]. The methods in this paper to study the Wilson polynomials are different from the method used in [25].

The structure of this paper is as follows. In Section 2 we define the affine Weyl group, construct a representation, and define a degenerate double affine Hecke algebra 
using this representation. The nonsymmetric Wilson polynomials are needed here to decompose our representation into irreducible representations. In Section 3 we study the nonsymmetric Wilson polynomials. We find orthogonality relations with respect to a complex measure, we obtain the duality property, and we relate the nonsymmetric Wilson polynomials to the well-known ${ }_{4} F_{3}$-polynomials defined by Wilson [24]. In Section 4 we define the polynomial Fourier transform as an integral transform with the nonsymmetric Wilson polynomials as a kernel. This Fourier transform maps polynomials to functions that are finitely supported on the polynomial spectrum $\Gamma$ of the Dunkl-Cherednik-type operator. The algebra $\mathcal{H}$ also acts on these functions, and we show that the Fourier transform intertwines actions of the algebra $\mathcal{H}$ on polynomials with the actions on functions on $\Gamma$. We also determine the inverse transform, and we give Plancherel-type formulas. Finally, in Section 5 we define and study a Fourier transform with a nonpolynomial kernel. We first introduce a Gaussian-type function. With this Gaussian, the polynomial Fourier transform and its inverse, we construct a Fourier transform that maps polynomials multiplied by the Gaussian to polynomials multiplied by another Gaussian. This Fourier transform also intertwines actions of the algebra $\mathcal{H}$. We show that we can write this Fourier transform as an integral transform with a nonsymmetric Wilson function as a kernel, by relating it to the Wilson function transform I. This nonpolynomial Fourier transform turns out to be self-dual.

Acknowledgment. I would like to thank Jasper Stokman and Genkai Zhang for helpful suggestions and comments.

\section{A degenerate double affine Hecke algebra}

In this section we define a representation of the affine Weyl group corresponding to the affine root system $\left(C_{1}^{\vee}, C_{1}\right)$. With this representation we construct a degenerate double affine Hecke algebra.

\subsection{The affine root system}

Let $E$ be the space of affine linear transformation of $\mathbb{R}$. Let $\delta$ be the constant function 1 , then for every $f \in E$ we may write

$$
f(u)=v u+\mu \delta(u), \quad u \in \mathbb{R},
$$

for some $v, \mu \in \mathbb{R}$, so we can identify $E$ with $\mathbb{R} \oplus \mathbb{R} \delta$. Let $\mathcal{R} \subset E$ be the subset

$$
\mathcal{R}=\left\{ \pm 1+\frac{1}{2} m \delta, \pm 2+m \delta \mid m \in \mathbb{Z}\right\} .
$$

The set $\mathcal{R}$ is the nonreduced affine root system of type $\left(C_{1}^{\vee}, C_{1}\right)$. Define a bilinear form $(\cdot, \cdot)$ on $E$ by

$$
\left(v_{1}+\mu_{1} \delta, v_{2}+\mu_{2} \delta\right)=v_{1} v_{2} .
$$

For every root $\alpha \in \mathcal{R}$ we define $s_{\alpha}: E \rightarrow E$ to be the reflection in the affine hyperplane $H_{\alpha}=\alpha^{-1}(0)$, then $s_{\alpha}$ is given by

$$
s_{\alpha}(f)=f-\left(f, \alpha^{\vee}\right) \alpha, \quad f \in E,
$$

where $\alpha^{\vee}=2 \alpha /(\alpha, \alpha)$. The affine Weyl group $\mathcal{W}$ is the group generated by the reflection $s_{\alpha}, \alpha \in \mathcal{R}$. 
Let $S=\{ \pm 2\} \subset \mathcal{R}$ and $S^{\vee}=\{ \pm 1\} \subset \mathcal{R}$. The subset $R=S \cup S^{\vee} \subset \mathcal{R}$ is a nonreduced (nonaffine) root system with Weyl group $W$ generated by the reflections $s_{\alpha}, \alpha \in R$. We set $a_{1}=2 \in \mathcal{R}$ and $a_{0}=\delta-2 \in \mathcal{R}$, then $a_{i}^{\vee}=a_{i} / 2, i=0,1$. Then the affine Weyl group $\mathcal{W}$ is the Coxeter group with two generators $s_{0}=s_{a_{0}}, s_{1}=s_{a_{1}}$, and relations $s_{i}^{2}=1$. The subgroup $W$ is generated by $s_{1}$, so $W=\left\{1, s_{1}\right\}$. Observe that $s_{1} s_{0} \in \mathcal{W}$ acts as a translation operator

$$
\left(s_{1} s_{0} f\right)(u)=f(u+1), \quad f \in E, u \in \mathbb{R} .
$$

Setting $\tau(\lambda)=\left(s_{1} s_{0}\right)^{\lambda} \in \mathcal{W}$ for $\lambda \in \mathbb{Z}$, we obtain a description of $\mathcal{W}$ as the semidirect product

$$
\mathcal{W}=W \ltimes \tau(\mathbb{Z}) .
$$

The affine root system $\mathcal{R}$ decomposes into four $\mathcal{W}$-orbits, namely

$$
\begin{array}{rlrl}
\mathcal{W} a_{0} & =S+(1+2 \mathbb{Z}) \delta, & \mathcal{W} a_{1}=S+2 \mathbb{Z} \delta, \\
\mathcal{W} a_{0}^{\vee}=S^{\vee}+\left(\frac{1}{2}+\mathbb{Z}\right) \delta, & \mathcal{W} a_{1}^{\vee}=S^{\vee}+\mathbb{Z} \delta .
\end{array}
$$

Let $\mathcal{S}=\mathcal{W} a_{0} \cup \mathcal{W} a_{1}$ and $\mathcal{S}^{\vee}=\mathcal{W} a_{0}^{\vee} \cup \mathcal{W} a_{1}^{\vee}$. Then $\mathcal{S}$ and $\mathcal{S}^{\vee}$ are reduced affine root systems with basis $\left\{a_{0}, a_{1}\right\}$, respectively, $\left\{a_{0}^{\vee}, a_{1}^{\vee}\right\}$, and $\mathcal{R}$ is the disjoint union $\mathcal{S} \cup \mathcal{S}^{\vee}$. Both $\mathcal{S}$ and $\mathcal{S}^{\vee}$ have $\mathcal{W}$ as corresponding affine Weyl group.

\subsection{The group algebra $\mathbb{C}[\mathcal{W}]$}

We extend the action of $\mathcal{W}$ on $E$ to an action of $\mathcal{W}$ on meromorphic functions on $\mathbb{C}$ by

$$
\left(s_{0} f\right)(x)=f(1-x), \quad\left(s_{1} f\right)(x)=f(-x) .
$$

In particular, this gives an action of $\mathcal{W}$ on the algebra $\mathcal{A}$ of polynomials in one variable. Using the expression $\tau(1)=s_{1} s_{0}$ it follows that $\tau(\lambda), \lambda \in \mathbb{Z}$, acts as the translation operator

$$
(\tau(\lambda) f)(x)=f(x+\lambda) .
$$

Let $\mathbf{t}$ be a multiplicity function, i.e., a function $\mathbf{t}: \mathcal{R} \rightarrow \mathbb{C}$ that is constant on $\mathcal{W}$ orbits. Since $\mathbf{t}$ is completely determined by the values $t_{a_{0}}, t_{a_{1}}, t_{a_{0}}$, and $t_{a_{1}^{\vee}}$, we may identify $\mathbf{t}$ with the ordered 4 -tuple $\left(t_{a_{0}}, t_{a_{0}^{\vee}}, t_{a_{1}}, t_{a_{1}}\right)$. Moreover, to ease notations we will write

$$
t_{a_{0}}=t_{0}, \quad t_{a_{0}^{\vee}}=u_{0}, t_{a_{1}}=t_{1}, t_{a_{1}^{\vee}}=u_{1} .
$$

For an object $O(\mathbf{t})$ (function, operator, etc.) depending on a multiplicity function $\mathbf{t}$ we will often suppress the dependence on $\mathbf{t}$ and we write $O(\mathbf{t})=O$.

Let $\mathbb{C}[\mathcal{W}]$ be the group algebra of the affine Weyl group $\mathcal{W}$. We assume $t_{0}, t_{1} \neq 0$. In $\mathbb{C}[\mathcal{W}]$ we define

$$
\sigma_{0}=t_{0} s_{0}, \quad \sigma_{1}=t_{1} s_{1}, \quad \xi=\sigma_{0}+\sigma_{1} .
$$

From $\left(\xi-\sigma_{i}\right)^{2}=t_{1-i}^{2}$ it follows that

$$
\xi^{2}=\sigma_{i} \xi+\xi \sigma_{i}+t_{1-i}^{2}-t_{i}^{2}, \quad i=0,1 .
$$


Now we see that $\xi^{2}$ commutes with $\sigma_{0}$ and $\sigma_{1}$. Moreover, iterating (2.1) for $i=1$ gives the relations

$$
\sigma_{1} \xi^{2 n}-\xi^{2 n} \sigma_{1}=0, \quad \sigma_{1} \xi^{2 n+1}+\xi^{2 n+1} \sigma_{1}=\left(\xi^{2}-t_{0}^{2}+t_{1}^{2}\right) \xi^{2 n}, \quad n \in \mathbb{N} .
$$

Let $\mathcal{A}_{\xi}$ denote the commutative subalgebra of $\mathbb{C}[\mathcal{W}]$ consisting of elements $p(\xi)$ for $p \in \mathcal{A}$. Then the above relations can be written as

$$
\sigma_{1} p(\xi)-\left(s_{1} p\right)(\xi) \sigma_{1}=\frac{t_{1}^{2}-t_{0}^{2}+\xi^{2}}{2 \xi}\left(p(\xi)-\left(s_{1} p\right)(\xi)\right)
$$

where $1 / \xi=\xi^{-1}$. Note that the right-hand side is an element of $\mathcal{A}_{\xi}$. This immediately leads to the following proposition.

Proposition 2.1. The sets $\left\{\xi^{m}, \xi^{n} \sigma_{1} \mid n, m \in \mathbb{Z}_{\geqslant 0}\right\}$ and $\left\{\xi^{m}, \sigma_{1} \xi^{n} \mid n, m \in \mathbb{Z}_{\geqslant 0}\right\}$ are linear bases for $\mathbb{C}[\mathcal{W}]$. So

$$
\mathcal{A}_{\xi} \otimes \mathbb{C}[W] \cong \mathbb{C}[\mathcal{W}] \cong \mathbb{C}[W] \otimes \mathcal{A}_{\xi}
$$

as vector spaces.

Let $\mathcal{A}^{W} \subset \mathcal{A}$ be the subalgebra of $W$-invariant polynomials, i.e., $\mathcal{A}^{W}=\mathbb{C}\left[x^{2}\right]$, and let $\mathcal{A}_{\xi}^{W} \subset \mathcal{A}_{\xi}$ be the subalgebra of $\mathbb{C}[\mathcal{W}]$ consisting of elements $p(\xi)$ for $p \in \mathcal{A}^{W}$. The subalgebra $\mathcal{A}_{\xi}^{W}$ is the center of $\mathbb{C}[\mathcal{W}]$. This can, for example, be checked using Proposition 2.1 and (2.2).

\subsection{A representation of $\mathbb{C}[\mathcal{W}]$}

To the simple roots $a_{0}, a_{1} \in \mathcal{S}$ we associate linear operators $T_{a_{i}}(\mathbf{t})=T_{i}$ by

$$
\left(T_{i} f\right)(x)=t_{i} f(x)+c_{i}(x)\left(\left(s_{i} f\right)(x)-f(x)\right),
$$

where $c_{i}$ are rational functions defined by

$$
c_{i}(x ; \mathbf{t})=\frac{\left(t_{i}+u_{i}+a_{i}^{\vee}(x)\right)\left(t_{i}-u_{i}+a_{i}^{\vee}(x)\right)}{a_{i}(x)} .
$$

Recall here that $a_{0}(x)=1-2 x$ and $a_{1}(x)=2 x$. It is easy to verify that the functions $c_{i}$ satisfy

$$
c_{i}(x)+\left(s_{i} c_{i}\right)(x)=2 t_{i}, \quad i=0,1 .
$$

Proposition 2.2. For $i=0,1$ the operators $T_{i}$ map $\mathcal{A}$ into itself.

To prove the proposition we introduce difference-reflection operators $D_{i}$ related to the simple roots $a_{i} \in \mathcal{S}$,

$$
D_{i}=\frac{s_{i}-1}{a_{i}} .
$$

Proposition 2.2 then follows immediately from the following lemma. 
Lemma 2.3. The operators $D_{i}, i=0,1$, map $\mathcal{A}$ into itself. Moreover, we have

$$
\begin{aligned}
D_{0} x^{m} & = \begin{cases}\frac{1}{2} m x^{m-2}+\text { l.o.t. }, & m \text { even }, \\
x^{m-1}-\frac{1}{2}(m-1) x^{m-2}+\text { l.o.t., } & \text { m odd },\end{cases} \\
D_{1} x^{m} & = \begin{cases}0, & m \text { even }, \\
-x^{m-1}, & m \text { odd. }\end{cases}
\end{aligned}
$$

Here "l.o.t." means "lower-order terms with respect to the degree".

Proof. By linearity it is enough the prove that $D_{i} p \in \mathcal{A}$ for $p(x)=x^{m}, m \in \mathbb{Z}_{\geqslant 0}$. For $i=1$ the calculation is straightforward. For $i=0$ we have

$$
D_{0} x^{m}=\frac{(1-x)^{m}-x^{m}}{1-2 x} \text {. }
$$

We replace $x$ by $z+\frac{1}{2}$, then we see that the numerator of the expression

$$
\frac{\left(\frac{1}{2}-z\right)^{m}-\left(\frac{1}{2}+z\right)^{m}}{-2 z}
$$

only contains odd powers of $z$, so we see that $D_{0} x^{m} \in \mathcal{A}$. To get the explicit expression we expand $\left(\frac{1}{2}+z\right)^{m}=\sum_{i=0}^{m}\left(\begin{array}{c}m \\ i\end{array}\right) z^{i} 2^{i-m}$ and write out the above expression.

Proposition 2.4. The assignment

$$
\sigma_{i} \mapsto T_{i}, \quad i=0,1,
$$

extends uniquely to an algebra homomorphism $\pi_{\mathbf{t}}: \mathbb{C}[\mathcal{W}] \rightarrow \operatorname{End}(\mathcal{A})$.

Proof. We only need to verify that $T_{i}$ satisfies the relations $T_{i}^{2}=t_{i}^{2}$. Let $p \in \mathcal{A}$, then we have, for $i=0,1$,

$$
\begin{aligned}
\left(T_{i}^{2} p\right)(x)= & T_{i}\left(t_{i} p(x)+c_{i}(x)\left(\left(s_{i} p\right)(x)-p(x)\right)\right) \\
= & t_{i}\left(t_{i} p(x)+c_{i}(x)\left(\left(s_{i} p\right)(x)-p(x)\right)\right) \\
& +c_{i}(x)\left(t_{i}\left(s_{i} p\right)(x)+\left(s_{i} c_{i}\right)(x)\left(p(x)-\left(s_{i} p\right)(x)\right)\right) \\
& -c_{i}(x)\left(t_{i} p(x)+c_{i}(x)\left(\left(s_{i} p\right)(x)-p(x)\right)\right) \\
= & \left(t_{i}^{2}+c_{i}(x)\left[c_{i}(x)+\left(s_{i} c_{i}\right)(x)-2 t_{i}\right]\right) p(x) \\
& -c_{i}(x)\left[c_{i}(x)+\left(s_{i} c_{i}\right)(x)-2 t_{i}\right]\left(s_{i} p\right)(x) .
\end{aligned}
$$

By (2.3) the terms between square brackets are equal to zero, hence $T_{i}^{2}=t_{i}^{2}$ as required.

We denote $\mathcal{T}=\pi_{\mathbf{t}}(\mathbb{C}[\mathcal{W}]) \subset \operatorname{End}(\mathcal{A})$, so $\mathcal{T}$ is the algebra generated by $T_{0}$ and $T_{1}$. Moreover, we will denote $T=\pi_{\mathbf{t}}(\mathbb{C}[W])$, so $T$ is the algebra generated by $T_{1}$. In the study of the representation $\pi_{\mathbf{t}}$ the image of $\xi \in \mathbb{C}[\mathcal{W}]$ will play an important role. We denote

$$
Y=\pi_{\mathbf{t}}(\xi)=T_{0}+T_{1} \in \mathcal{T} \text {. }
$$

For $Y$ we have the following useful property. 
Proposition 2.5. $Y$ is a triangular operator, i.e., for $m \in \mathbb{Z}_{\geqslant 0}$,

$$
Y x^{m}=\gamma_{m} x^{m}+\text { l.o.t. }
$$

The coefficients $\gamma_{m}$ are given explicitly by

$$
\gamma_{m}= \begin{cases}t_{0}+t_{1}+n, & m=2 n, \\ -\left(t_{0}+t_{1}+n\right), & m=2 n-1 .\end{cases}
$$

From here on we assume that $t_{0}+t_{1} \notin-\frac{1}{2} \mathbb{N}$, so that $\gamma_{m_{1}} \neq \gamma_{m_{2}}$ if $m_{1} \neq m_{2}$.

Proof. We write

$$
\begin{aligned}
& T_{0}=t_{0}+\left(t_{0}+u_{0}+\frac{1}{2}-x\right)\left(t_{0}-u_{0}+\frac{1}{2}-x\right) D_{0}, \\
& T_{1}=t_{1}+\left(t_{1}+u_{1}+x\right)\left(t_{1}-u_{1}+x\right) D_{1},
\end{aligned}
$$

then we obtain, from Lemma 2.3,

$$
\begin{aligned}
& T_{0} x^{m}= \begin{cases}x^{m+1}-\left(t_{0}+\frac{1}{2}(m+1)\right) x^{m}+\text { l.o.t., } & m \text { odd }, \\
\left(t_{0}+\frac{1}{2} m\right) x^{m}+\text { l.o.t., } & m \text { even, }\end{cases} \\
& T_{1} x^{m}= \begin{cases}-x^{m+1}-t_{1} x^{m}+\text { l.o.t., } & m \text { odd, } \\
t_{1} x^{m}+\text { l.o.t. }, & m \text { even. }\end{cases}
\end{aligned}
$$

Now the result follows from $Y=T_{0}+T_{1}$.

From Proposition 2.5 it follows that for all $m \in \mathbb{Z}_{\geqslant 0}$ there exists, up to a multiplicative constant, a unique element in $\mathcal{A}$ which is an eigenfunction of $Y$ for eigenvalue $\gamma_{m}$. We call this element a nonsymmetric Wilson polynomial.

Definition 2.6. The nonsymmetric Wilson polynomial $p_{m}$ is the unique monic polynomial of degree $m$ such that $Y p_{m}=\gamma_{m} p_{m}$.

Clearly the set $\left\{p_{m} \mid m \in \mathbb{Z}_{\geqslant 0}\right\}$ forms a linear basis for $\mathcal{A}$. In order to decompose $\mathcal{A}$ as a $\mathcal{T}$-module, we need to know the action of $T_{1}$ on the nonsymmetric Wilson polynomials. By $T_{0}=Y-T_{1}$ we then also have the action of $T_{0}$.

Proposition 2.7. We have $T_{1} p_{0}=t_{1} p_{0}$ and, for $m \geqslant 1$,

$$
T_{1} p_{m}= \begin{cases}-p_{m+1}+b_{m} p_{m}, & m \text { odd }, \\ b_{m} p_{m}+\left(b_{m}^{2}-t_{1}^{2}\right) p_{m-1}, & m \text { even }\end{cases}
$$

where

$$
b_{m}=\frac{\left(\gamma_{m}+t_{1}+t_{0}\right)\left(\gamma_{m}+t_{1}-t_{0}\right)}{2 \gamma_{m}}-t_{1}
$$

If we define $b_{0}=t_{1}$, which is consistent with the above-defined expression for $b_{m}$, then the expression for $T_{1} p_{m}, m$ even, is also valid for $m=0$ if we assume that $p_{-1}$ exists (e.g., $p_{-1}=1$ ). 
Proof. From $p_{0}=1$ we obtain directly $T_{1} p_{0}=t_{1} p_{0}$. Assume $m \geqslant 1$. From the action of $T_{1}$ on the monomial $x^{m}$ (see the proof of Proposition 2.5) it follows that

$$
T_{1} p_{2 n-1}=-p_{2 n}+\sum_{j=0}^{2 n-1} a_{2 n-1, j} p_{j}, \quad T_{1} p_{2 n}=\sum_{j=0}^{2 n} a_{2 n, j} p_{j}, \quad n \in \mathbb{N}
$$

Now using $Y T_{1}+T_{1} Y=Y^{2}+t_{1}^{2}-t_{0}^{2}$ we find $\left(Y+\gamma_{m}\right) T_{1} p_{m}=\left(\gamma_{m}^{2}+t_{1}^{2}-t_{0}^{2}\right) p_{m}$. Applying this to the expansions gives

$$
a_{m, m}=\frac{\gamma_{m}^{2}+t_{1}^{2}-t_{0}^{2}}{2 \gamma_{m}}, \quad a_{2 n-1,2 n-2}=0, \quad a_{m, j}=0, \quad j \leqslant m-2
$$

Note that we do not find the value for $a_{2 n, 2 n-1}$ from this, since $\gamma_{2 n-1}+\gamma_{2 n}=0$. In order to find $a_{2 n, 2 n-1}$ we write out $T_{1}^{2} p_{2 n}$ :

$$
\begin{aligned}
T_{1}^{2} p_{2 n} & =T_{1}\left(a_{2 n, 2 n} p_{2 n}+a_{2 n, 2 n-1} p_{2 n-1}\right) \\
& =\left(a_{2 n, 2 n}^{2}-a_{2 n, 2 n-1}\right) p_{2 n}+a_{2 n, 2 n-1}\left(a_{2 n, 2 n}+a_{2 n-1,2 n-1}\right) p_{2 n-1}
\end{aligned}
$$

Using $\gamma_{2 n}=-\gamma_{2 n-1}$ we see that $a_{2 n, 2 n}+a_{2 n-1,2 n-1}=0$. Now from $T_{1}^{2}=t_{1}^{2}$ it follows that $\left(a_{2 n, 2 n}^{2}-a_{2 n, 2 n-1}\right)=t_{1}^{2}$, which gives us the value of $a_{2 n, 2 n-1}$.

We denote by $\mathcal{A}_{Y} \subset \mathcal{T}$ and $\mathcal{A}_{Y}^{W} \subset \mathcal{T}$ the commutative subalgebras consisting of elements $p(Y)$ for $p \in \mathcal{A}$, respectively, $p \in \mathcal{A}^{W}$. Also, we set $\mathcal{A}(n)=\operatorname{span}\left\{p_{2 n}, p_{2 n-1}\right\}$, $n \in \mathbb{N}$, and $\mathcal{A}(0)=\operatorname{span}\left\{p_{0}\right\}$.

\section{Theorem 2.8.}

(a) The representation $\pi_{\mathbf{t}}: \mathbb{C}[\mathcal{W}] \rightarrow \operatorname{End}(\mathcal{A})$ is faithful.

(b) The decomposition $\mathcal{A}=\bigoplus_{n \in \mathbb{Z}_{\geqslant 0}} \mathcal{A}(n)$ is the multiplicity-free, irreducible decomposition of $\mathcal{A}$ as a $\mathbb{C}[\mathcal{W}]$-module. Moreover, the element $p(\xi)$ in the center $\mathcal{A}_{\xi}^{W}$ of $\mathbb{C}[\mathcal{W}]$ acts on $\mathcal{A}(n)$ as multiplication by $p\left(\gamma_{2 n}\right)$.

Proof. (a) By Proposition 2.1 every element of $\mathcal{T}=\pi_{\mathbf{t}}(\mathbb{C}[\mathcal{W}])$ can be written as $p(Y)+$ $T_{1} q(Y)$ for some $p, q \in \mathcal{A}$. Suppose that $p(Y)+T_{1} q(Y)=0$ in $\mathcal{T}$, then $\left(p\left(\gamma_{m}\right)+\right.$ $\left.q\left(\gamma_{m}\right) T_{1}\right) p_{m}=0$ for all $m \in \mathbb{Z}_{\geqslant 0}$. Now it follows from Proposition 2.7 that $q\left(\gamma_{m}\right)=0$ for all $m$, hence $q=0$ in $\mathcal{A}$. This leaves us with $p\left(\gamma_{m}\right)=0$ for all $m$, so also $p=0$ in $\mathcal{A}$.

(b) The decomposition follows from Proposition 2.7 and the fact that the algebra $\mathcal{T}$ is generated by $T_{1}$ and $Y$. Let $p(\xi) \in \mathcal{A}_{\xi}^{W}$, then since $\gamma_{2 n}=-\gamma_{2 n-1}$ we find that $\pi_{\mathbf{t}}(p(\xi))=p(Y)$ acts on $\mathcal{A}(n)=\operatorname{span}\left\{p_{2 n-1}, p_{2 n}\right\}$ as multiplication by $p\left(\gamma_{2 n-1}\right)=$ $p\left(\gamma_{2 n}\right)$.

\subsection{A degenerate double affine Hecke algebra}

Let $z \in \operatorname{End}(\mathcal{A})$ be multiplication by $x ;(z p)(x)=x p(x)$ for $p \in \mathcal{A}$. We denote by $\mathcal{A}_{z}$ the commutative algebra in $\operatorname{End}(\mathcal{A})$ of multiplication operators $p(z)$ for $p \in \mathcal{A}$. Let us now define a degenerate double affine Hecke algebra as follows. 
Definition 2.9. The algebra $\mathcal{H}=\mathcal{H}(\mathbf{t})$ is the algebra in $\operatorname{End}(\mathcal{A})$ generated by $\mathcal{T}$ and by $\mathcal{A}_{z}$.

From writing the explicit expressions $T_{i}, i=0,1$, as

$$
T_{i}=t_{i} s_{i}+\frac{t_{i}^{2}-u_{i}^{2}+\left(a_{i}^{\vee}(\cdot)\right)^{2}}{a_{i}(\cdot)}\left(s_{i}-1\right),
$$

it follows that we have the following relations in $\mathcal{H}$,

$$
T_{i} p(z)-\left(s_{i} p\right)(z) T_{i}=\frac{t_{i}^{2}-u_{i}^{2}+\left(a_{i}^{\vee}(z)\right)^{2}}{a_{i}(z)}\left(\left(s_{i} p\right)(z)-p(z)\right) .
$$

Using the linear bases for $\mathbb{C}[\mathcal{W}]$ from Proposition 2.1 we obtain linear bases for $\mathcal{H}$.

Proposition 2.10. The sets

$$
\left\{Y^{k} z^{l}, Y^{m} T_{1} z^{n} \mid k, l, m, n \in \mathbb{Z}_{\geqslant 0}\right\}, \quad\left\{z^{k} Y^{l}, z^{m} T_{1} Y^{n} \mid k, l, m, n \in \mathbb{Z}_{\geqslant 0}\right\},
$$

are linear bases for $\mathcal{H}$. So

$$
\mathcal{A}_{Y} \otimes T \otimes \mathcal{A}_{z} \cong \mathcal{H} \cong \mathcal{A}_{z} \otimes T \otimes \mathcal{A}_{Y}
$$

as vector spaces.

Now we can show that the relations (2.4) and the quadratic relations in $\mathcal{T}$ completely characterize the algebra $\mathcal{H}$.

Proposition 2.11. The algebra $\mathcal{H}(\mathbf{t})$ is isomorphic to the unital, associative algebra $\mathcal{V}(\mathbf{t})$ generated by $V_{0}, V_{1}, v$ with relations

$$
V_{i}^{2}=t_{i}^{2}, \quad i=0,1,
$$

and, for $p \in \mathcal{A}$,

$$
V_{i} p(v)-\left(s_{i} p\right)(v) V_{i}=\frac{t_{i}^{2}-u_{i}^{2}+\left(a_{i}^{\vee}(v)\right)^{2}}{a_{i}(v)}\left(\left(s_{i} p\right)(v)-p(v)\right),
$$

The isomorphism $\phi: \mathcal{V}(\mathbf{t}) \rightarrow \mathcal{H}(\mathbf{t})$ is given on generators by the assignments

$$
V_{0} \mapsto T_{0}, \quad V_{1} \mapsto T_{1}, \quad v \mapsto z .
$$

Proof. Let $\phi$ be defined on the generators of $\mathcal{V}$ as in the proposition, then by $T_{i}^{2}=t_{i}^{2}$, $i=0,1$, and by $(2.4), \phi$ preserves the defining relations of $\mathcal{V}$. Moreover, $\phi$ maps generators of $\mathcal{V}$ to generators of $\mathcal{H}$, hence $\phi$ is surjective. For the injectivity it is enough to show that the set

$$
B=\left\{\left(V_{0}+V_{1}\right)^{k} z^{l},\left(V_{0}+V_{1}\right)^{m} V_{1} z^{n} \mid k, l, m, n \in \mathbb{Z}_{\geqslant 0}\right\}
$$

is a linear basis for $\mathcal{V}$. Now observe that the relations $V_{i}^{2}=t_{i}^{2}$ imply that the subalgebra generated by $V_{0}$ and $V_{1}$ is isomorphic to $\mathbb{C}[\mathcal{W}]$, then from Proposition 2.1 and the other relations in $\mathcal{V}$ it follows that the set $B$ is indeed a basis for $\mathcal{V}$. 
For $p(z)=z \in \mathcal{A}_{z}$ the relations (2.4) become

$$
\begin{aligned}
T_{0} z+z T_{0}-T_{0} & =t_{0}^{2}-u_{0}^{2}+\left(\frac{1}{2}-z\right)^{2}, \\
T_{1} z+z T_{1} & =u_{1}^{2}-t_{1}^{2}-z^{2},
\end{aligned}
$$

which we can also write as

$$
\left(T_{0}+\frac{1}{2}-z\right)^{2}=u_{0}^{2}, \quad\left(T_{1}+z\right)^{2}=u_{1}^{2}
$$

Let us therefore denote

$$
T_{a_{0}^{\vee}}=-T_{0}-\frac{1}{2}+z, \quad T_{a_{1}^{\vee}}=-T_{1}-z,
$$

then we have operators associated to any simple root in $\mathcal{R}$ such that

$$
T_{\alpha}^{2}=t_{\alpha}^{2}, \quad \alpha \in\left\{a_{0}, a_{1}, a_{0}^{\vee}, a_{1}^{\vee}\right\}
$$

We will also use the notation $T_{a_{i}^{\vee}}=U_{i}, i=0,1$. Using Proposition 2.11 we now obtain the following characterization of the algebra $\mathcal{H}$.

Proposition 2.12. The algebra $\mathcal{H}(\mathbf{t})$ is isomorphic to the unital, associative algebra $\mathcal{V}(\mathbf{t})$ generated by $V_{i}, \widetilde{V}_{i}, i=0,1$, with relations

$$
\begin{gathered}
V_{i}^{2}=t_{i}^{2}, \quad \widetilde{V}_{i}^{2}=u_{i}^{2}, \\
V_{0}+V_{1}+\widetilde{V}_{0}+\widetilde{V}_{1}=-\frac{1}{2} .
\end{gathered}
$$

The isomorphism is given explicitly on generators by $V_{i} \mapsto T_{i}, \widetilde{V}_{i} \mapsto U_{i}$ for $i=0,1$.

Proof. The last relation in the proposition implies that $\mathcal{V}$ is also generated as an algebra by the elements $V_{0}, V_{1}$ and $v=-V_{1}-\widetilde{V}_{1}=\frac{1}{2}+V_{0}+\widetilde{V}_{0}$. Writing out the quadratic relations $\widetilde{V}_{i}^{2}=u_{i}^{2}$ leads to

$$
\begin{aligned}
V_{0} v+v V_{0}-V_{0} & =t_{0}^{2}-u_{0}^{2}+\left(\frac{1}{2}-v\right)^{2}, \\
V_{1} v+v V_{1} & =u_{1}^{2}-t_{1}^{2}-v^{2} .
\end{aligned}
$$

Iterating these relations gives back the defining relations from Proposition 2.11.

Finally, to justify the name "degenerate double affine Hecke algebra" for our algebra $\mathcal{H}$ we show that the operators $T_{0}$ and $T_{1}$ can be obtained by taking an appropriate limit in the Noumi representation of the affine Hecke algebra of type $\tilde{A}_{1}$.

Let $0<q<1$ and let $\mathbf{k}: \mathcal{R} \rightarrow \mathbb{C}$ be a multiplicity function. The affine Hecke algebra of type $\tilde{A}_{1}$ is the unital complex algebra generated by $V_{0}$ and $V_{1}$ with relations

$$
\left(V_{j}-k_{a_{j}}\right)\left(V_{j}+k_{a_{j}}^{-1}\right)=0, \quad j=0,1 .
$$

The Noumi representation is given by

$$
V_{j}=k_{a_{j}}+k_{a_{j}}^{-1} \frac{\left(1-k_{a_{j}} k_{a_{j}^{\vee}} q^{a_{j}^{\vee}(x)}\right)\left(1-k_{a_{j}} k_{a_{j}^{\vee}}^{-1} q^{a_{j}^{\vee}(x)}\right)}{1-q^{a_{j}(x)}}\left(s_{j}-1\right) .
$$


These operators act on the algebra of Laurent polynomials in $q^{x}$. Now we substitute

$$
\left(k_{a_{0}}, k_{a_{0}^{\vee}}, k_{a_{1}}, k_{a_{1}^{\vee}}\right) \mapsto\left(-i q^{t_{0}}, i q^{u_{0}},-i q^{t_{1}}, i q^{u_{1}}\right),
$$

with $t_{0}, u_{0}, t_{1}, u_{1} \in \mathbb{C}$, then

$$
\lim _{q \uparrow 1} \frac{1-i V_{j}}{1-q}=t_{j}+\frac{\left(t_{j}+u_{j}+a_{j}^{\vee}(x)\right)\left(t_{j}-u_{j}+a_{j}^{\vee}(x)\right)}{a_{j}(x)}\left(s_{j}-1\right)=T_{j}, \quad j=0,1 .
$$

So the algebra $\mathcal{T}$ generated by $T_{0}$ and $T_{1}$ is indeed a degenerate affine Hecke algebra. Moreover, the Dunkl-Cherednik operator for the affine Hecke algebra is given by $\tilde{Y}=$ $V_{1} V_{0}$. In the limit we obtain

$$
\lim _{q \uparrow 1} \frac{\tilde{Y}+1}{1-q}=\lim _{q \uparrow 1}\left(\frac{1-i V_{0}}{1-q}+\frac{1-i V_{1}}{1-q}-\frac{\left(1-i V_{0}\right)\left(1-i V_{1}\right)}{1-q}\right)=T_{0}+T_{1}=Y .
$$

The algebra $\mathcal{H}$ may be considered as a $q=1$ analogue of the double affine Hecke algebra of type $\left(C_{1}^{\vee}, C_{1}\right)$, which was introduced by Sahi [20] for general rank. The presentation of $\mathcal{H}$ in Proposition 2.12 corresponds to Stokman's characterization [18, Theorem 2.22] of Sahi's double affine Hecke algebra.

The algebra $\mathcal{H}$ has appeared earlier in the literature. I would like to thank the referee for pointing this out. Consider the affine Dynkin quiver $Q$ of type $\tilde{D}_{4}$ with corresponding deformed preprojective algebra $\Pi_{Q}^{\lambda}$, see [6] for the definitions. Let $i_{0}, i_{1}, \ldots, i_{4}$ be the vertices in $Q$, where $i_{0}$ is the nodal vertex with associated idempotent $e_{0}$. The spherical subalgebra $e_{0} \Pi_{Q}^{\lambda} e_{0}$ is generated by $X_{1}, \ldots, X_{4}$ with relations

$$
\begin{aligned}
X_{k}\left(X_{k}-\lambda_{i_{k}}\right) & =0, \quad k=1, \ldots, 4, \\
X_{1}+X_{2}+X_{3}+X_{4} & =\lambda_{i_{0}},
\end{aligned}
$$

see [7, Theorem 7.2], [16, Theorem 1]. Now take

$$
\lambda=\left(\lambda_{i_{0}}, \lambda_{i_{1}}, \lambda_{i_{2}}, \lambda_{i_{3}}, \lambda_{i_{4}}\right)=\left(t_{0}+u_{0}+t_{1}+u_{1}-\frac{1}{2}, 2 t_{0}, 2 u_{0}, 2 t_{1}, 2 u_{1}\right),
$$

then it follows from Proposition 2.12 that the assignments

$$
X_{1} \mapsto T_{0}+t_{0}, \quad X_{2} \mapsto U_{0}+u_{0}, \quad X_{3} \mapsto T_{1}+t_{1}, \quad X_{4} \mapsto U_{1}+u_{1},
$$

extend to an algebra isomorphism $e_{0} \Pi_{Q}^{\lambda} e_{0} \rightarrow \mathcal{H}(\mathbf{t})$. It is shown in [7] that, for appropriate $\lambda$, the algebra $e_{0} \Pi_{Q}^{\lambda} e_{0}$ is a rational degeneration of the double affine Hecke algebra of type $\left(C_{1}^{\vee}, C_{1}\right)$.

\section{Wilson polynomials}

In this section we study the nonsymmetric Wilson polynomials from Definition 2.6, and their symmetrized versions. We show that the symmetric Wilson polynomials are (with a suitable normalization) exactly the Wilson polynomials as defined by Wilson in $[24]$. 


\subsection{Orthogonality relations}

Let us introduce parameters $a, b, c, d$ related to the multiplicity function $\mathbf{t}=\left(t_{0}, u_{0}, t_{1}, u_{1}\right)$ by

$$
(a, b, c, d)=\left(t_{1}+u_{1}, t_{1}-u_{1}, t_{0}+u_{0}+\frac{1}{2}, t_{0}-u_{0}+\frac{1}{2}\right) .
$$

Throughout the rest of the paper, the parameters $a, b, c, d$ will be related to the multiplicity function $\mathbf{t}$ in this way. We define a weight function $\Delta$ by

$$
\Delta(x ; \mathbf{t})=\frac{\Gamma(a+x) \Gamma(a+1-x) \Gamma(b+x) \Gamma(b+1-x) \Gamma(c \pm x) \Gamma(d \pm x)}{\Gamma(2 x) \Gamma(1-2 x)} .
$$

Here, and elsewhere, we use the notation $f(\alpha \pm \beta)=f(\alpha+\beta) f(\alpha-\beta)$. From here on we assume that the multiplicity function $\mathbf{t}$ is such that:

- $a, b, c, d \notin-\frac{1}{2} \mathbb{Z}_{\geqslant 0}$;

- the pairwise sum of $a, b, c, d$ is not contained in $\mathbb{Z}$.

Let $\mathcal{C}=\mathcal{C}_{\mathbf{t}}$ be a contour in the complex plane that runs along the imaginary axis from $-i \infty$ to $i \infty$ and is indented such that the sequences $a+n, b+n, c+n, d+n, n \in \mathbb{Z} \geqslant 0$, are separated by $\mathcal{C}$ from the sequences $-(a+n),-(b+n),-(c+n),-(d+n), n \in \mathbb{Z}_{\geqslant 0}$. Moreover, we assume that, set theoretically, $\mathcal{C}=-\mathcal{C}$. With the above assumptions on t such a contour exists. To the weight function $\Delta$ we now associate a nondegenerate bilinear form $\langle\cdot, \cdot\rangle_{\mathbf{t}}$ on $\mathcal{A}$,

$$
\langle f, g\rangle_{\mathbf{t}}=\frac{1}{2 \pi i} \int_{\mathcal{C}} f(x) g(x) \Delta(x) d x .
$$

By Cauchy's theorem we may write the above integral as an integral over $i \mathbb{R}$ plus a finite sum of residues corresponding to poles of $\Delta$. In case $a, b, c, d$ have positive real part we may take $\mathcal{C}=i \mathbb{R}$.

Proposition 3.1. The operators $Y$ and $T_{i}, i=0,1$, are symmetric with respect to $\langle\cdot, \cdot\rangle_{\mathbf{t}}$.

Proof. Let $f, g \in \mathcal{A}$. We have

$$
\left(T_{0} f\right)(x)=t_{0} f(x)+\frac{(c-x)(d-x)}{1-2 x}(f(1-x)-f(x)),
$$

SO

$$
\left\langle T_{0} f, g\right\rangle_{\mathbf{t}}=\frac{1}{2 \pi i} \int_{\mathcal{C}} t_{0} f(x) g(x) \Delta(x) d x+\frac{1}{2 \pi i} \int_{\mathcal{C}}(f(1-x)-f(x)) g(x) \hat{\Delta}(x) d x,
$$

where

$$
\begin{aligned}
& \hat{\Delta}(x) \\
& \quad=\frac{\Gamma(a+x) \Gamma(a+1-x) \Gamma(b+x) \Gamma(b+1-x) \Gamma(c+x) \Gamma(c+1-x) \Gamma(d+x) \Gamma(d+1-x)}{\Gamma(2 x) \Gamma(2-2 x)} .
\end{aligned}
$$


We see that $\hat{\Delta}(x)=\hat{\Delta}(1-x)$. So we may write the second integral as

$$
\frac{1}{2 \pi i} \int_{1+\mathcal{C}} f(x) g(1-x) \hat{\Delta}(x) d x-\frac{1}{2 \pi i} \int_{\mathcal{C}} f(x) g(x) \hat{\Delta}(x) d x
$$

Under the current assumptions on $\mathbf{t}$ the integrand of the first integral does not have poles inside the area between $\mathcal{C}$ and $1+\mathcal{C}$. Therefore we can shift the path of integration, and we obtain

$$
\begin{aligned}
\left\langle T_{0} f, g\right\rangle_{\mathbf{t}} & =\frac{1}{2 \pi i} \int_{\mathcal{C}} f(x)\left(t_{0} g(x)+\frac{(c-x)(d-x)}{1-2 x}(g(1-x)-g(x))\right) \Delta(x) d x \\
& =\left\langle f, T_{0} g\right\rangle_{\mathbf{t}}
\end{aligned}
$$

This proves the proposition for $T_{0}$. The proof for $T_{1}$ is similar. Since $Y=T_{0}+T_{1}, Y$ is also symmetric with respect to $\langle\cdot, \cdot\rangle_{\mathbf{t}}$.

We can now prove the orthogonality relations for the nonsymmetric Wilson polynomials.

Theorem 3.2. The set of nonsymmetric Wilson polynomials $\left\{p_{m} \mid m \in \mathbb{Z}_{\geqslant 0}\right\}$ is an orthogonal basis for $\mathcal{A}$ with respect to $\langle\cdot, \cdot\rangle_{\mathbf{t}}$.

Proof. Using the previous proposition we have

$$
\gamma_{m_{1}}\left\langle p_{m_{1}}, p_{m_{2}}\right\rangle_{\mathbf{t}}=\left\langle Y p_{m_{1}}, p_{m_{2}}\right\rangle_{\mathbf{t}}=\left\langle p_{m_{1}}, Y p_{m_{2}}\right\rangle_{\mathbf{t}}=\gamma_{m_{2}}\left\langle p_{m_{1}}, p_{m_{2}}\right\rangle_{\mathbf{t}}
$$

Since $\gamma_{m_{1}} \neq \gamma_{m_{2}}$ if $m_{1} \neq m_{2}$, we have

$$
\left\langle p_{m_{1}}, p_{m_{2}}\right\rangle_{\mathbf{t}}=0
$$

if $m_{1} \neq m_{2}$.

We evaluate the diagonal terms $\left\langle p_{m}, p_{m}\right\rangle_{\mathbf{t}}$ later on in Theorem 4.3.

Remark 3.3. We could also define a bilinear form by

$$
\langle f, g\rangle_{\mathbf{t}}^{\prime}=\frac{1}{2 \pi i} \int_{\mathcal{C}} f(x) g(-x) \Delta(x) d x .
$$

This bilinear form is closer to the bilinear form used for the nonsymmetric Askey-Wilson polynomials in [18]. However, $T_{0}$ and $T_{1}$ are not symmetric with respect to this bilinear form. So this would lead to biorthogonality relations between the eigenfunctions of $Y$ and $Y^{*}$, the adjoint of $Y$ with respect to the above-defined bilinear form. However, it is not hard to show that the eigenfunctions of $Y^{*}$ are precisely the nonsymmetric Wilson polynomials $x \mapsto p_{m}(-x$; $\mathbf{t})$, so that this biorthogonality relation is equivalent to the orthogonality relations in Theorem 3.2. 


\subsection{Symmetric and antisymmetric Wilson polynomials}

Let us define in $\mathbb{C}[W]$,

$$
c_{ \pm}=\frac{1}{2}\left(1 \pm s_{1}\right) .
$$

These elements are orthogonal primitive idempotents in $\mathbb{C}[W]$, i.e., $c_{ \pm}^{2}=c_{ \pm}, c_{-}+c_{+}=1$, and $c_{ \pm} c_{\mp}=0$. For $p \in \mathcal{A}$ the polynomial $c_{+} p$ is an even polynomial, so $c_{+}$is the projection of $\mathcal{A}$ onto $\mathcal{A}^{W}$, and $c_{-} p$ is an odd polynomial. The representation $\pi_{\mathbf{t}}$ of $\mathbb{C}[W]$ gives us corresponding elements in the algebra $T$,

$$
C_{ \pm}=\pi_{\mathbf{t}}\left(c_{ \pm}\right)=\frac{1}{2 t_{1}}\left(t_{1} \pm T_{1}\right) \in T .
$$

Since $C_{+}+C_{-}=1$ in $T$, we have a corresponding decomposition of $\mathcal{A}$ in a symmetric and an antisymmetric part; $\mathcal{A}=\mathcal{A}_{+} \oplus \mathcal{A}_{-}$, where $\mathcal{A}_{ \pm}=C_{ \pm} \mathcal{A}$. So $\mathcal{A}_{ \pm}$consists of polynomials $p \in \mathcal{A}$ for which $\left(T_{1} \mp t_{1}\right) p=0$. From the explicit expression of $T_{1}$ we obtain $T_{1} p=t_{1} p$ if and only if $s_{1} p=p$, so we see that $\mathcal{A}_{+}=\mathcal{A}^{W}$, the algebra of even polynomials. Moreover, $\mathcal{A}_{-}$consists of the polynomials $p$ such that $c_{1}(-x) p(x)$ is odd. Indeed, the identity $\left(T_{1}+t_{1}\right) p=0$ is equivalent to

$$
0=2 t_{1} p(x)+c_{1}(x)(p(-x)-p(x))=\left(c_{1}(x)+c_{1}(-x)\right) p(x)+c_{1}(x)(p(-x)-p(x)),
$$

which gives $c_{1}(-x) p(x)=-c_{1}(x) p(-x)$. The symmetric and the antisymmetric polynomials are related by the generalized Weyl denominator, which is the monic antisymmetric polynomial of lowest degree.

Proposition 3.4. Let the generalized Weyl denominator $\delta$ be the polynomial given by

$$
\delta(x)=\left(t_{1}+u_{1}+x\right)\left(t_{1}-u_{1}+x\right)
$$

then $\delta(z) \mathcal{A}_{+}=\mathcal{A}_{-}$.

Proof. After a straightforward calculation it follows from Proposition 2.11 that

$$
\left(T_{1}+t_{1}\right) \delta(z)=\delta(-z)\left(T_{1}-t_{1}\right),
$$

hence $\delta(z) \mathcal{A}_{+} \subset \mathcal{A}_{-}$. In the same way it follows that $\delta(z)^{-1} \mathcal{A}_{-}$is $W$-invariant. So we only need to show that if $p \in \mathcal{A}_{-}$, then $\delta(x)^{-1} p(x)$ is a polynomial. This follows directly from writing the polynomial $p \in \mathcal{A}_{-}$as

$$
p(x)=-\frac{c_{1}(x)}{c_{1}(-x)} p(-x)=\frac{\delta(x)}{\delta(-x)} p(-x),
$$

where we used $\delta(x)=2 x c_{1}(x)$. Hence $\delta(z)^{-1} \mathcal{A}_{-} \subset \mathcal{A}_{+}$.

We can decompose the irreducible $\mathcal{T}$-modules in a symmetric and antisymmetric part; $\mathcal{A}(n)=\mathcal{A}_{+}(n) \oplus \mathcal{A}_{-}(n), n \in \mathbb{Z}_{\geqslant 0}$. So the nonsymmetric Wilson polynomials $p_{m}$ can be written as the sum of a symmetric and an antisymmetric polynomial. For a factor $F_{n}^{ \pm}$independent of $x$, we must have $C_{ \pm} p_{2 n-1}=F_{n}^{ \pm} C_{ \pm} p_{2 n}$, since both $C_{ \pm} p_{2 n}$ and $C_{ \pm} p_{2 n-1}$ are in the (anti-)symmetric part of $\mathcal{A}(n)$. It will be useful to work with monic polynomials. 


\section{Definition 3.5.}

(a) The symmetric Wilson polynomial $P_{2 n}^{+}$is the unique monic polynomial in $\mathcal{A}_{+}(n)$.

(b) The antisymmetric Wilson polynomial $P_{2 n}^{-}$is the unique monic polynomial in $\mathcal{A}_{-}(n)$.

We remark that both $P_{2 n}^{+}$and $P_{2 n}^{-}$are of degree $2 n$. There are no (anti)symmetric Wilson polynomials of odd degree. Note that the sets $\left\{P_{2 n}^{+} \mid n \in \mathbb{Z}_{\geqslant 0}\right\}$ and $\left\{P_{2 n}^{-} \mid n \in \mathbb{N}\right\}$ are a linear basis for $\mathcal{A}_{+}$, respectively, $\mathcal{A}_{-}$. We can express the (anti)symmetric Wilson polynomials in terms of nonsymmetric ones and vice versa.

Lemma 3.6. We have

$$
P_{2 n}^{+}=p_{2 n}+\left(b_{2 n}-t_{1}\right) p_{2 n-1}, \quad P_{2 n}^{-}=p_{2 n}+\left(b_{2 n}+t_{1}\right) p_{2 n-1},
$$

or, equivalently,

$$
p_{2 n}=\frac{1}{2 t_{1}}\left(\left(b_{2 n}+t_{1}\right) P_{2 n}^{+}-\left(b_{2 n}-t_{1}\right) P_{2 n}^{-}\right), \quad p_{2 n-1}=\frac{1}{2 t_{1}}\left(P_{2 n}^{-}-P_{2 n}^{+}\right),
$$

with $b_{2 n}$ as in Proposition 2.7 .

Proof. This follows from writing out $\left(T_{1} \pm t_{1}\right) p_{m}$ using Proposition 2.7.

The (anti)symmetric Wilson polynomials also satisfy orthogonality relations with respect to the bilinear form $\langle\cdot, \cdot\rangle_{\mathbf{t}}$.

Lemma 3.7. We have the following orthogonality relations:

(a) For $n \in \mathbb{Z}_{\geqslant 0}, m \in \mathbb{N},\left\langle P_{2 n}^{+}, P_{2 m}^{-}\right\rangle_{\mathbf{t}}=0$;

(b) For $n, m \in \mathbb{Z}_{\geqslant 0}, n \neq m,\left\langle P_{2 n}^{+}, P_{2 m}^{+}\right\rangle_{\mathbf{t}}=0$;

(c) For $n, m \in \mathbb{N}, n \neq m,\left\langle P_{2 n}^{-}, P_{2 m}^{-}\right\rangle_{\mathbf{t}}=0$.

Proof. (a) From $C_{ \pm} C_{\mp}=0$ we obtain

$$
\left\langle C_{+} p_{n}, C_{-} p_{m}\right\rangle_{\mathbf{t}}=\left\langle p_{n}, C_{+} C_{-} p_{m}\right\rangle_{\mathbf{t}}=0 .
$$

(b) Since $P_{2 n}^{+} \in \mathcal{A}^{W}$ and $Y^{2} \in \mathcal{A}_{Y}^{W}$, it follows from Theorem 2.8(b) that $Y^{2} P_{2 n}^{+}=$ $\gamma_{2 n}^{2} P_{2 n}^{+}$. Because $Y$ is symmetric with respect to $\langle\cdot, \cdot\rangle_{\mathbf{t}}, Y^{2}$ is also symmetric. Now, since the eigenvalues $\gamma_{2 n}^{2}$ are pairwise different, the orthogonality relations follow.

(c) Theorem 3.2 gives, for $n \neq m$,

$0=\left\langle p_{n}, p_{m}\right\rangle_{\mathbf{t}}=\left\langle C_{+} p_{n}, C_{+} p_{m}\right\rangle_{\mathbf{t}}+\left\langle C_{+} p_{n}, C_{-} p_{m}\right\rangle_{\mathbf{t}}+\left\langle C_{-} p_{n}, C_{+} p_{m}\right\rangle_{\mathbf{t}}+\left\langle C_{-} p_{n}, C_{-} p_{m}\right\rangle_{\mathbf{t}}$.

Then by (a) and (b) we obtain $\left\langle C_{-} p_{n}, C_{-} p_{m}\right\rangle_{\mathbf{t}}=0$.

Let us define the weight function $\Delta^{+}$by

$$
\Delta^{+}(x ; \mathbf{t})=\frac{\Gamma(a \pm x) \Gamma(b \pm x) \Gamma(c \pm x) \Gamma(d \pm x)}{\Gamma( \pm 2 x)},
$$

and let $\langle\cdot, \cdot\rangle_{\mathbf{t}}^{+}$be the corresponding bilinear form on $\mathcal{A}_{+}$,

$$
\langle f, g\rangle_{\mathbf{t}}^{+}=\frac{1}{2 \pi i} \int_{\mathcal{C}} f(x) g(x) \Delta^{+}(x) d x .
$$

Observe that $\Delta^{+}(-x)=\Delta^{+}(x)$. 
Lemma 3.8. For $f, g \in \mathcal{A}_{+}$,

$$
\langle f, g\rangle_{\mathbf{t}}=\frac{1}{2}(a+b)\langle f, g\rangle_{\mathbf{t}}^{+} .
$$

Proof. From the explicit expression for $\Delta$ and $\Delta^{+}$it follows that

$$
\Delta(x)=c_{1}(-x) \Delta^{+}(x) .
$$

Let $f, g \in \mathcal{A}_{+}$, then

$$
2\langle f, g\rangle_{\mathbf{t}}=\frac{1}{2 \pi i} \int_{\mathcal{C}} f(x) g(x)\left(c_{1}(x)+c_{1}(-x)\right) \Delta^{+}(x) d x .
$$

Then the result follows from $c_{1}(x)+c_{1}(-x)=2 t_{1}=a+b$.

Combining Lemma 3.8 with Lemma 3.7(b) then leads to the orthogonality relations for the symmetric Wilson polynomials with respect to $\langle\cdot, \cdot\rangle_{\mathbf{t}}^{+}$.

Theorem 3.9. The set $\left\{P_{2 n}^{+} \mid n \in \mathbb{Z}_{\geqslant 0}\right\}$ is an orthogonal basis for $\mathcal{A}_{+}$with respect to $\langle\cdot, \cdot\rangle_{\mathbf{t}}^{+}$.

Now we can prove the generalized Weyl character formula, which says that antisymmetric Wilson polynomials can be expressed in terms of symmetric Wilson polynomials with a shift in the parameters using the generalized Weyl denominator $\delta$ defined in Proposition 3.4.

Theorem 3.10. For $n \in \mathbb{N}$,

$$
P_{2 n}^{-}(x ; \mathbf{t})=\delta(x) P_{2 n-2}^{+}\left(x ; t_{0}, u_{0}, t_{1}+1, u_{1}\right) .
$$

Proof. The symmetric Wilson polynomial $P_{2 n}^{+}\left(\cdot ; t_{0}, u_{0}, t_{1}+1, u_{1}\right)$ is the unique even monic polynomial of degree $2 n$ that is orthogonal to all even polynomials of degree $\leqslant 2 n-2$ with respect to $\langle\cdot, \cdot\rangle_{\left(t_{0}, u_{0}, t_{1}+1, u_{1}\right)}^{+}$. Let $p(x)=\delta(x)^{-1} P_{2 n}^{-}(x ; \mathbf{t})$, then $p$ is a monic polynomial and by Proposition 3.4 we have $p \in \mathcal{A}_{+}$. So, to prove the desired identity, it is enough to show that $\left\langle p, q_{2 k}\right\rangle_{\left(t_{0}, u_{0}, t_{1}+1, u_{1}\right)}^{+}=0$, for any even polynomial $q_{2 k}$ of degree $2 k$ for $k=0, \ldots, n-2$.

By the explicit expressions for $\Delta$ and $\Delta^{+}$we have

$$
\delta(x) \Delta(x ; \mathbf{t})=-\frac{1}{2 x} \Delta^{+}\left(x ; t_{0}, u_{0}, t_{1}+1, u_{1}\right) .
$$

Now we write out the bilinear form $\langle\cdot, \cdot\rangle_{\mathbf{t}}$ as an integral and we symmetrize the integrand, then we obtain

$$
\begin{aligned}
\left\langle P_{2 n}^{-}, \delta(z) q_{2 k}\right\rangle_{\mathbf{t}} & =\frac{1}{4 \pi i} \int_{\mathcal{C}} p(x) q_{2 k}(x)\left(\frac{\delta(-x)-\delta(x)}{2 x}\right) \Delta^{+}\left(x ; t_{0}, u_{0}, t_{1}+1, u_{1}\right) d x \\
& =-\frac{1}{2}(a+b)\left\langle p, q_{2 k}\right\rangle_{\left(t_{0}, u_{0}, t_{1}+1, u_{1}\right)}^{+} .
\end{aligned}
$$

Since $\delta(z) q_{2 k} \in \operatorname{span}\left\{P_{2 m}^{-} \mid m=1, \ldots, k+1\right\} \subset \mathcal{A}_{-}$it follows from Lemma 3.7(c) that $\left\langle P_{2 n}^{-}, \delta(z) q_{2 k}\right\rangle_{\mathbf{t}}=0$, hence $\left\langle p, q_{2 k}\right\rangle_{\left(t_{0}, u_{0}, t_{1}+1, u_{1}\right)}^{+}=0$ as desired. 


\subsection{Duality}

In this subsection we prove the duality property for the Wilson polynomials using ideas from [20] and [18]. We define an involution $\sigma$ acting on multiplicity functions by interchanging the values on the $a_{0}$-orbit and the $a_{1}^{\vee}$-orbit. So, given a multiplicity function $\mathbf{t}=\left(t_{0}, u_{0}, t_{1}, u_{1}\right)$, the multiplicity function $\mathbf{t}^{\sigma}: \mathcal{R} \rightarrow \mathbb{C}$ is given by

$$
\mathbf{t}^{\sigma}=\left(t_{a_{0}}^{\sigma}, t_{a_{0}}^{\sigma}, t_{a_{1}}^{\sigma}, t_{a_{1}}^{\sigma}\right)=\left(u_{1}, u_{0}, t_{1}, t_{0}\right) .
$$

We call $\mathbf{t}^{\sigma}$ the dual of $\mathbf{t}$. If an object depends on the multiplicity function $\mathbf{t}$, we will use a super- or subscript $\sigma$ to denote the same object depending on $\mathbf{t}^{\sigma}$. For instance, for the difference-reflection operators we write $T_{i}^{\sigma}=T_{i}\left(\mathbf{t}^{\sigma}\right)$, and $\mathcal{H}_{\sigma}=\mathcal{H}\left(\mathbf{t}^{\sigma}\right)$ is the algebra generated by $T_{0}^{\sigma}, T_{1}^{\sigma}$ and $z^{\sigma}=z$.

Proposition 3.11. The assignments

$$
T_{0} \mapsto-\left(T_{1}^{\sigma}+z\right), \quad T_{1} \mapsto T_{1}^{\sigma}, \quad z \mapsto-\left(T_{0}^{\sigma}+T_{1}^{\sigma}\right)
$$

extend uniquely to an algebra isomorphism $\sigma: \mathcal{H} \rightarrow \mathcal{H}_{\sigma}$ with inverse $\sigma^{-1}=\sigma_{\sigma}$, respectively, anti-isomorphism $\psi: \mathcal{H} \rightarrow \mathcal{H}_{\sigma}$ with inverse $\psi^{-1}=\psi_{\sigma}$.

We call $\sigma$ and $\psi$, the duality isomorphism, respectively, duality anti-isomorphism.

Proof. It is straightforward to verify that the following relations are satisfied

$$
\sigma\left(T_{0}\right)^{2}=t_{0}^{2}, \quad \sigma\left(T_{1}\right)^{2}=t_{1}^{2}, \quad \sigma\left(z-\frac{1}{2}-T_{0}\right)^{2}=u_{0}^{2}, \quad \sigma\left(-T_{1}-z\right)^{2}=u_{1}^{2},
$$

and similarly for $\psi$. We see that both $\psi$ and $\sigma$ satisfy

$$
T_{0} \mapsto U_{1}^{\sigma}, \quad T_{1} \mapsto T_{1}^{\sigma}, \quad U_{0} \mapsto U_{0}^{\sigma}, \quad U_{1} \mapsto T_{0}^{\sigma}, \quad z \mapsto-Y^{\sigma}, \quad Y \mapsto-z
$$

We then see that $\sigma^{-1}=\sigma_{\sigma}$ and $\psi^{-1}=\psi_{\sigma}$.

Next we introduce, following Sahi [20], elements in $\mathcal{H}$ called intertwiners which can be used to construct raising and lowering operators for the nonsymmetric Wilson polynomials. These intertwiners $S_{0}, S_{1} \in \mathcal{H}$ are defined by

$$
S_{0}=U_{1} Y-Y U_{1}, \quad S_{1}=T_{1} Y-Y T_{1} .
$$

We have the following useful property.

Lemma 3.12. For $n \in \mathbb{Z}_{\geqslant 0}$,

$$
S_{0} p_{2 n}=\left(\gamma_{2 n+1}-\gamma_{2 n}\right) p_{2 n+1}, \quad S_{1} p_{2 n+1}=2 \gamma_{2 n+2} p_{2 n+2} .
$$

Proof. By Proposition 2.7 we have

$$
S_{1} p_{2 n+1}=\left(\gamma_{2 n+1}-Y\right) T_{1} p_{2 n+1}=\left(\gamma_{2 n+1}-Y\right)\left(-p_{2 n+2}+b_{2 n+1} p_{2 n+1}\right) .
$$

Then the formula for $S_{1}$ follows from $\gamma_{2 n+2}=-\gamma_{2 n+1}$.

Next we check the action of $S_{0}$. Let $\widetilde{S}_{0}=z T_{0}-T_{0} z \in \mathcal{H}$. For $p \in \mathcal{A}$, we obtain from Proposition $2.11 p(z) \widetilde{S}_{0}=\widetilde{S}_{0}\left(s_{0} p\right)(z)$ in $\mathcal{H}$. Applying the duality isomorphism $\sigma$ and replacing the parameters by their duals, gives us $p(-Y) S_{0}=S_{0} p(1+Y)$ in $\mathcal{H}$. So $S_{0} p_{2 n}$ is an eigenfunction of $Y$ for eigenvalue $-\left(1+\gamma_{2 n}\right)=\gamma_{2 n+1}$, therefore $S_{0} p_{2 n}=k_{n} p_{2 n+1}$ for some constant $k_{n}$. Using Proposition 2.7 again, the constant $k_{n}$ can be determined by finding the leading coefficient in

$$
S_{0} p_{2 n}=\left(Y-\gamma_{2 n}\right)\left(T_{1}+z\right) p_{2 n}=\left(Y-\gamma_{2 n}\right)\left(\left(b_{2 n}+z\right) p_{2 n}+\left(b_{2 n}^{2}-t_{1}^{2}\right) p_{2 n-1}\right) .
$$

From Proposition 2.5 it now follows that $k_{n}=\gamma_{2 n+1}-\gamma_{2 n}$. 
Observe that in the same way as in Lemma 3.12 it can be proved that $S_{0} p_{2 n+1}=$ $k_{n}^{0} p_{2 n}$ and $S_{1} p_{2 n}=k_{n}^{1} p_{2 n-1}$ for some constants $k_{n}^{i}$ which can be determined explicitly. We do not need these formulas here.

As a first application of Lemma 3.12 we deduce a Rodriguez-type formula for the nonsymmetric Wilson polynomials, which says that the nonsymmetric Wilson polynomials can be generated from $1 \in \mathcal{A}$, the polynomial identically equal to 1 , using the intertwiners. We use the standard notation for shifted factorials,

$$
(\alpha)_{0}=1, \quad(\alpha)_{n}=\alpha(\alpha+1) \ldots(\alpha+n-1), \quad n \in \mathbb{N} .
$$

Proposition 3.13. For $n \in \mathbb{Z}_{\geqslant 0}$,

$$
\begin{aligned}
\left(S_{1} S_{0}\right)^{n} 1 & =(-1)^{n}\left(2 t_{0}+2 t_{1}+1\right)_{2 n} p_{2 n}, \\
S_{0}\left(S_{1} S_{0}\right)^{n} 1 & =(-1)^{n+1}\left(2 t_{0}+2 t_{1}+1\right)_{2 n+1} p_{2 n+1} .
\end{aligned}
$$

Proof. This follows from Lemma 3.12 using induction on the degree.

As a second application of Lemma 3.12 we evaluate $p_{m}\left(-x_{0}\right)$ explicitly, where $x_{0}=$ $t_{1}+u_{1}$. For this we introduce the evaluation mapping Ev $: \mathcal{H} \rightarrow \mathbb{C}$ by

$$
\operatorname{Ev}(X)=(X(1))\left(-x_{0}\right), \quad X \in \mathcal{H},
$$

where $1 \in \mathcal{A}$. From the explicit expression $T_{1}=t_{1}+c_{1}(\cdot)\left(s_{1}-1\right)$ and from $c_{1}\left(-x_{0}\right)=0$ it follows directly that

$$
\operatorname{Ev}\left(T_{1} X\right)=t_{1} \operatorname{Ev}(X), \quad X \in \mathcal{H}
$$

Proposition 3.14. For $n \in \mathbb{N}$,

$$
\begin{aligned}
p_{2 n}\left(-x_{0}\right) & =\frac{(a+b+1)_{n}(a+c)_{n}(a+d)_{n}}{(n+a+b+c+d)_{n}}, \\
p_{2 n-1}\left(-x_{0}\right) & =-\frac{(a+b+1)_{n-1}(a+c)_{n}(a+d)_{n}}{(n+a+b+c+d-1)_{n}} \\
P_{2 n}^{+}\left(x_{0}\right) & =\frac{(a+b)_{n}(a+c)_{n}(a+d)_{n}}{(n+a+b+c+d-1)_{n}} .
\end{aligned}
$$

Proof. Let $n \in \mathbb{Z}_{\geqslant 0}$. Using the relation $Y T_{1}+T_{1} Y=Y^{2}+t_{1}^{2}-t_{0}^{2}$ in $\mathcal{H}$, we may write

$$
S_{1}=2 T_{1} Y-Y^{2}-t_{1}^{2}+t_{0}^{2} .
$$

Then using (3.3) we have

$$
\begin{aligned}
\operatorname{Ev}\left(S_{1} p_{2 n+1}(z)\right) & =\left(2 t_{1} \gamma_{2 n+1}-\gamma_{2 n+1}^{2}-t_{1}^{2}+t_{0}^{2}\right) p_{2 n+1}\left(-x_{0}\right) \\
& =\left(t_{0}+t_{1}-\gamma_{2 n+1}\right)\left(t_{0}-t_{1}+\gamma_{2 n+1}\right) p_{2 n+1}\left(-x_{0}\right) .
\end{aligned}
$$

Applying the duality isomorphism $\sigma$ to the relation $T_{0} z+z T_{0}-T_{0}=t_{0}^{2}-u_{0}^{2}+\left(\frac{1}{2}-z\right)^{2}$ and replacing the parameters by their duals we find, in $\mathcal{H}$,

$$
U_{1} Y+Y U_{1}+U_{1}=u_{0}^{2}-u_{1}^{2}-\left(\frac{1}{2}+Y\right)^{2} .
$$


This gives us

$$
S_{0}=-2\left(T_{1}+z\right) Y-\left(T_{1}+z\right)+\left(\frac{1}{2}+Y\right)^{2}+u_{1}^{2}-u_{0}^{2}
$$

and using (3.3) we then obtain

$$
\begin{aligned}
\operatorname{Ev}\left(S_{0} p_{2 n}(z)\right) & =\left(-2\left(t_{1}-x_{0}\right) \gamma_{2 n}-\left(t_{1}-x_{0}\right)+\left(\frac{1}{2}+\gamma_{2 n}\right)^{2}+u_{1}^{2}-u_{0}^{2}\right) p_{2 n}\left(-x_{0}\right) \\
& =\left(u_{1}+u_{0}+\frac{1}{2}+\gamma_{2 n}\right)\left(u_{1}-u_{0}+\frac{1}{2}+\gamma_{2 n}\right) p_{2 n}\left(-x_{0}\right) .
\end{aligned}
$$

On the other hand, we find from Lemma 3.12,

$$
\operatorname{Ev}\left(S_{0} p_{2 n}(z)\right)=\left(\gamma_{2 n+1}-\gamma_{2 n}\right) p_{2 n+1}\left(-x_{0}\right), \quad \operatorname{Ev}\left(S_{1} p_{2 n+1}(z)\right)=2 \gamma_{2 n+2} p_{2 n+2}\left(-x_{0}\right)
$$

Combining now gives us the recurrence relations

$$
\begin{aligned}
& p_{2 n+1}\left(-x_{0}\right)=-\frac{\left(t_{0}+t_{1}+u_{0}+u_{1}+\frac{1}{2}+n\right)\left(t_{0}+t_{1}-u_{0}+u_{1}+\frac{1}{2}+n\right)}{2 t_{0}+2 t_{1}+2 n+1} p_{2 n}\left(-x_{0}\right), \\
& p_{2 n+2}\left(-x_{0}\right)=-\frac{\left(2 t_{0}+2 t_{1}+1+n\right)\left(2 t_{1}+1+n\right)}{2 t_{0}+2 t_{1}+2 n+2} p_{2 n+1}\left(-x_{0}\right) .
\end{aligned}
$$

The evaluation formula for $p_{m}\left(-x_{0}\right)$ follows by induction on $m$, starting with $p_{0}\left(-x_{0}\right)=$ 1. The expression for $P_{2 n}^{+}\left(x_{0}\right)=P_{2 n}^{+}\left(-x_{0}\right)$ follows from Lemma 3.6.

Similar to Ev we define the dual evaluation mapping $\widetilde{\mathrm{Ev}}: \mathcal{H}_{\sigma} \rightarrow \mathbb{C}$ by

$$
\widetilde{\operatorname{Ev}}(\tilde{X})=(\widetilde{X}(1))\left(-\gamma_{0}\right), \quad \tilde{X} \in \mathcal{H}_{\sigma}
$$

With the evaluation mappings and the duality anti-isomorphism $\psi$ we construct two pairings $B: \mathcal{H} \times \mathcal{H}_{\sigma} \rightarrow \mathbb{C}$ and $\widetilde{B}: \mathcal{H}_{\sigma} \times \mathcal{H} \rightarrow \mathbb{C}$ as follows:

$$
B(X, \widetilde{X})=\operatorname{Ev}\left(\psi_{\sigma}(\widetilde{X}) X\right), \quad \widetilde{B}(\widetilde{X}, X)=\widetilde{\operatorname{Ev}}(\psi(X) \widetilde{X}), \quad X \in \mathcal{H}, \tilde{X} \in \mathcal{H}_{\sigma} .
$$

These pairings have the following properties.

Lemma 3.15. Let $X, X_{1}, X_{2} \in \mathcal{H}$ and $\widetilde{X}, \widetilde{X}_{1}, \widetilde{X}_{2} \in \mathcal{H}_{\sigma}$, and let $p \in \mathcal{A}$. Then:

(a) $B(X, \widetilde{X})=\widetilde{B}(\widetilde{X}, X)$;

(b) $B\left(X_{1} X_{2}, \widetilde{X}\right)=B\left(X_{2}, \psi\left(X_{1}\right) \widetilde{X}\right)$, and $B\left(X, \widetilde{X}_{1} \widetilde{X}_{2}\right)=B\left(\psi_{\sigma}\left(\widetilde{X}_{1}\right) X, \widetilde{X}_{2}\right)$;

(c) $B((X p)(z), \widetilde{X})=B(X p(z), \widetilde{X})$, and $B(X,(\widetilde{X} p)(z))=B(X, \widetilde{X} p(z))$.

Proof. (a) Let $X=f(z) T_{1} g(Y) \in \mathcal{H}$ for $f, g \in \mathcal{A}$, then using $Y 1=\gamma_{0}$ and $T_{1} 1=t_{1}$ we have

$$
\begin{aligned}
\widetilde{\operatorname{Ev}}(\psi(X)) & =\left(g(-z) T_{1}^{\sigma} f\left(-Y^{\sigma}\right)(1)\right)\left(-\gamma_{0}\right) \\
& =t_{1} f\left(-x_{0}\right) g\left(\gamma_{0}\right) \\
& =\left(f(z) T_{1} g(Y)(1)\right)\left(-x_{0}\right) \\
& =\operatorname{Ev}(X),
\end{aligned}
$$


and similarly for $X=f(z) g(Y)$. So we have $\widetilde{\operatorname{Ev}}(\psi(X))=\operatorname{Ev}(X)$ for all $X \in \mathcal{H}$. This gives us

$$
\widetilde{B}(\widetilde{X}, X)=\widetilde{\operatorname{Ev}}\left(\psi(X) \psi\left(\psi_{\sigma}(\widetilde{X})\right)\right)=\operatorname{Ev}\left(\psi_{\sigma}(\widetilde{X}) X\right)=B(X, \widetilde{X}) .
$$

(b) Since $\psi$ is an anti-isomorphism with inverse $\psi_{\sigma}$, we have

$$
B\left(X_{1} X_{2}, \widetilde{X}\right)=\operatorname{Ev}\left(\psi_{\sigma}(\widetilde{X}) X_{1} X_{2}\right)=\operatorname{Ev}\left(\psi_{\sigma}\left(\psi\left(X_{1}\right) \widetilde{X}\right) X_{2}\right)=B\left(X_{2}, \psi\left(X_{1}\right) \widetilde{X}\right) .
$$

(c) This is an immediate consequence of $((X p)(z))(1)=(X p)=X(p(z)(1))$ in $\mathcal{A}$.

We renormalize the nonsymmetric Wilson polynomials as follows:

$$
E\left(x, \gamma_{m} ; \mathbf{t}\right)=\frac{p_{m}(x ; \mathbf{t})}{p_{m}\left(-x_{0} ; \mathbf{t}\right)} .
$$

In particular, we have $\operatorname{Ev}\left(E\left(z, \gamma_{m}\right)\right)=1$ for all $m \in \mathbb{Z}_{\geqslant 0}$. Furthermore, we denote the eigenvalues of $Y^{\sigma} \in \mathcal{H}_{\sigma}$ by $x_{m}$, i.e., for $m \in \mathbb{Z}_{\geqslant 0}$,

$$
x_{m}=\gamma_{m}^{\sigma}= \begin{cases}t_{1}+u_{1}+n, & m=2 n, \\ -\left(t_{1}+u_{1}+n\right), & m=2 n-1 .\end{cases}
$$

We are now ready to prove the duality property for the nonsymmetric Wilson polynomials.

Theorem 3.16. For $m, n \in \mathbb{Z}_{\geqslant 0}$ and $p \in \mathcal{A}$, we have

$$
p\left(-\gamma_{m}\right)=\widetilde{B}\left(p(z), E\left(z, \gamma_{m}\right)\right), \quad p\left(-x_{n}\right)=B\left(p(z), E_{\sigma}\left(z, x_{n}\right)\right) .
$$

Consequently, the Wilson polynomials satisfy the duality property

$$
E\left(-x_{n}, \gamma_{m} ; \mathbf{t}\right)=E\left(-\gamma_{m}, x_{n} ; \mathbf{t}^{\sigma}\right) .
$$

Proof. Using $\operatorname{Ev}\left(E\left(z, \gamma_{m}\right)\right)=1$ for all $m \in \mathbb{Z}_{\geqslant 0}$ and the previous lemma we have, for $f \in \mathcal{A}$,

$$
\begin{aligned}
\widetilde{B}\left(f(z), E\left(z, \gamma_{m}\right)\right) & =\widetilde{B}\left(1, f(-Y) E\left(z, \gamma_{m}\right)\right) \\
& =\widetilde{B}\left(1,\left(f(-Y) E\left(\cdot, \gamma_{m}\right)\right)(z)\right) \\
& =f\left(-\gamma_{m}\right) \widetilde{B}\left(1, E\left(z, \gamma_{m}\right)\right) \\
& =f\left(-\gamma_{m}\right) .
\end{aligned}
$$

Similarly, for $g \in \mathcal{A}$, we find

$$
B\left(g(z), E_{\sigma}\left(z, x_{n}\right)\right)=g\left(-x_{n}\right) .
$$

Now take $f=E_{\sigma}\left(\cdot ; x_{n}\right)$ and $g=E\left(\cdot, \gamma_{m}\right)$, and use Lemma 3.15(a) to obtain the second statement of the theorem.

Theorem 3.16 can be used to write the actions of $T_{1}$ and $U_{1}$ on the Wilson polynomials as difference-reflection operators in the dual variable. This will be useful in the next section. 
Lemma 3.17. For $m \in \mathbb{Z}_{\geqslant 0}$

$$
\begin{aligned}
& \left(T_{1} E\left(\cdot ; \gamma_{m}\right)\right)(x)=t_{1} E\left(x ; \gamma_{m}\right)+c_{1}^{\sigma}\left(-\gamma_{m}\right)\left(E\left(x ;-\gamma_{m}\right)-E\left(x ; \gamma_{m}\right)\right), \\
& \left(U_{1} E\left(\cdot ; \gamma_{m}\right)\right)(x)=u_{1} E\left(x ; \gamma_{m}\right)+c_{0}^{\sigma}\left(-\gamma_{m}\right)\left(E\left(x ;-1-\gamma_{m}\right)-E\left(x ; \gamma_{m}\right)\right),
\end{aligned}
$$

where we use the convention $E\left(x,-\gamma_{0}\right)=1$.

It is useful to observe that $c_{1}^{\sigma}\left(-\gamma_{0}\right)=0$.

Proof. For $U_{1}$ we recall that, by Theorem 3.16,

$$
\left(U_{1} E\left(\cdot, \gamma_{m}\right)\right)\left(-x_{n}\right)=B\left(E\left(z, \gamma_{m}\right), T_{0}^{\sigma} E_{\sigma}\left(z, x_{n}\right)\right)
$$

Now we use the relations between $T_{0}$ and $p(z)$ in $\mathcal{H}$ as well as the identity $B\left(X, \tilde{X} T_{0}^{\sigma}\right)=$ $u_{1} B(X, \widetilde{X})$ for $X \in \mathcal{H}, \widetilde{X} \in \mathcal{H}_{\sigma}$, then we obtain, for $m, n \in \mathbb{Z}_{\geqslant 0}$,

$$
\begin{aligned}
& \left(U_{1} E\left(\cdot, \gamma_{m}\right)\right)\left(-x_{n}\right)=u_{1} B\left(E\left(z, \gamma_{m}\right), E_{\sigma}\left(1-z, x_{n}\right)\right) \\
& \quad+\frac{u_{1}^{2}-u_{0}^{2}+\left(\frac{1}{2}+\gamma_{m}\right)^{2} / 4}{1+2 \gamma_{m}}\left(B\left(E\left(z, \gamma_{m}\right), E_{\sigma}\left(1-z, x_{n}\right)\right)-B\left(E\left(z, \gamma_{m}\right), E_{\sigma}\left(z, x_{n}\right)\right)\right) \\
& \quad=u_{1} E\left(-x_{n}, \gamma_{m}\right)+c_{0}\left(-\gamma_{m} ; \mathbf{t}^{\sigma}\right)\left(E\left(-x_{n},-1-\gamma_{m}\right)-E\left(-x_{n}, \gamma_{m}\right)\right) .
\end{aligned}
$$

Here we used

$$
\begin{aligned}
B\left(E\left(z, \gamma_{m}\right), E_{\sigma}\left(1-z, x_{n}\right)\right) & =\widetilde{B}\left(E_{\sigma}\left(1-z, x_{n}\right), E\left(z, \gamma_{m}\right)\right) \\
& =E_{\sigma}\left(1+\gamma_{m}, x_{n}\right)=E\left(-x_{n},-1-\gamma_{m}\right) .
\end{aligned}
$$

So the desired identity holds for all $n \in \mathbb{Z}_{\geqslant 0}$, hence it holds in $\mathcal{A}$. The expression for $T_{1}$ can be obtained in the same way.

Let us also briefly consider the symmetric Wilson polynomials. We will denote $C_{ \pm} E\left(\cdot, \gamma_{m}\right)=E^{ \pm}\left(\cdot, \gamma_{m}\right)$. For $m=2 n$ or $m=2 n-1$ we have $C_{+} E\left(\cdot, \gamma_{m}\right)=k P_{2 n}^{+}$ for some nonzero constant $k$. From $c_{1}\left(-x_{0}\right)=0$ and $E\left(-x_{0}, \gamma_{m}\right)=1$ it follows that $\left(T_{1} E\left(\cdot, \gamma_{m}\right)\right)\left(-x_{0}\right)=t_{1}$. Therefore, $\left(C_{+} E\left(\cdot, \gamma_{m}\right)\right)\left(-x_{0}\right)=1$ which gives

$$
E^{+}\left(x, \gamma_{2 n} ; \mathbf{t}\right)=E^{+}\left(x, \gamma_{2 n-1} ; \mathbf{t}\right)=\frac{P_{2 n}^{+}(x ; \mathbf{t})}{P_{2 n}^{+}\left(x_{0} ; \mathbf{t}\right)}, \quad n \in \mathbb{N} .
$$

In the same way as in Theorem 3.16 the duality property for the renormalized symmetric Wilson polynomials is obtained;

$$
E^{+}\left(x_{2 n}, \gamma_{2 m} ; \mathbf{t}\right)=E^{+}\left(\gamma_{2 m}, x_{2 n} ; \mathbf{t}^{\sigma}\right), \quad m, n \in \mathbb{Z}_{\geqslant 0} .
$$

\subsection{Explicit expressions for the Wilson polynomials}

From the definition of $T_{0}$ and $T_{1}$, and from the description of $\mathcal{W}$ as the semidirect product $W \ltimes \tau(\mathbb{Z})$, it follows that we can write any $X \in \mathcal{T}$ as

$$
X=\sum_{\substack{\lambda \in \mathbb{Z} \\ w \in W}} c_{\lambda, w} \tau(\lambda) w
$$

for some coefficients $c_{\lambda, w} \in \mathbb{C}(x)$ of which only finitely many are nonzero. Since $W=$ $\left\{1, s_{1}\right\}$, we may write any $X \in \mathcal{T}$ as $X=X_{0}+X_{1} s_{1}$, where $X_{0}, X_{1} \in \bigoplus_{\lambda \in \mathbb{Z}} \mathbb{C}(x) \tau(\lambda)$. So $X_{0}$ and $X_{1}$ are difference operators with rational coefficients. We define $X_{\text {sym }}=X_{0}+X_{1}$. Since $\mathcal{A}_{+}$is $W$-invariant, it is clear the actions of $X$ and $X_{\text {sym }}$ on $\mathcal{A}_{+}$coincide.

We use the difference operator $\left(Y^{2}\right)_{\text {sym }}$ to obtain a difference equation for the symmetric Wilson polynomials. 


\section{Proposition 3.18.}

(a) The symmetric Wilson polynomials satisfy the equation

$$
L E^{+}\left(\cdot, \gamma_{2 n}\right)=n(n+a+b+c+d-1) E^{+}\left(\cdot, \gamma_{2 n}\right),
$$

where $L$ is the second-order difference operator

$$
\begin{gathered}
L=\left(Y^{2}\right)_{\mathrm{sym}}-\left(t_{0}+t_{1}\right)^{2}=A(x)(\tau(1)-1)+A(-x)(\tau(-1)-1), \\
A(x)=\frac{(a+x)(b+x)(c+x)(d+x)}{2 x(2 x+1)} .
\end{gathered}
$$

(b) The symmetric Wilson polynomials satisfy the recurrence relation

$$
\begin{aligned}
\left(x^{2}-a^{2}\right) E^{+}\left(x, \gamma_{2 n}\right)= & B_{n}\left(E^{+}\left(x, \gamma_{2 n+2}\right)-E^{+}\left(x, \gamma_{2 n}\right)\right) \\
& +C_{n}\left(E^{+}\left(x, \gamma_{2 n-2}\right)-E^{+}\left(x, \gamma_{2 n}\right)\right),
\end{aligned}
$$

where

$$
\begin{aligned}
B_{n} & =\frac{(n+a+b+c+d-1)(n+a+b)(n+a+c)(n+a+d)}{(2 n+a+b+c+d-1)(2 n+a+b+c+d)}, \\
C_{n} & =\frac{n(n+b+c-1)(n+b+d-1)(n+c+d-1)}{(2 n+a+b+c+d-2)(2 n+a+b+c+d-1)} .
\end{aligned}
$$

Here we use the convention $E^{+}\left(x, \gamma_{-2}\right)=0$.

Proof. (a) Since $E^{+}\left(\cdot, \gamma_{2 n}\right) \in \mathcal{A}(n)$ it follows from Theorem 2.8 that $Y^{2} E^{+}\left(\cdot, \gamma_{2 n}\right)=$ $\gamma_{2 n}^{2} E^{+}\left(\cdot, \gamma_{2 n}\right)$. Then, because $E^{+}\left(\cdot, \gamma_{2 n}\right) \in \mathcal{A}_{+}$, the symmetric Wilson polynomial $E^{+}\left(\cdot, \gamma_{2 n}\right)$ is a solution of the difference equation $\left(Y^{2}\right)_{\mathrm{sym}} f=\gamma_{2 n}^{2} f$. Now write

$$
\gamma_{2 n}^{2}-\left(t_{0}+t_{1}\right)^{2}=\left(t_{0}+t_{1}+n\right)^{2}-\left(t_{0}+t_{1}\right)^{2}=n\left(2 t_{0}+2 t_{1}+n\right),
$$

to see that $E^{+}\left(x, \gamma_{2 n}\right)$ is an eigenfunction of $L=\left(Y^{2}\right)_{\mathrm{sym}}-\left(t_{0}+t_{1}\right)^{2}$ for eigenvalue $n(n+a+b+c+d-1)$.

Now let us obtain the explicit expression for $\left(Y^{2}\right)_{\text {sym }}$. From the explicit expression for $T_{0}$ and $T_{1}$,

$$
T_{i}=t_{i}+c_{i}(\cdot)\left(s_{i}-1\right),
$$

and from $s_{0}=\tau(-1) s_{1}=s_{1} \tau(1)$ we see that we can write

$$
\left(Y^{2}\right)_{\mathrm{sym}}=B(x)[\tau(1)-1]+C(x)[\tau(-1)-1]+D(x)
$$

for $B, C, D \in \mathbb{C}(x)$. To find $D$ it is enough to calculate $Y^{2} 1$. From $T_{i} 1=t_{i}$, since $1 \in \mathcal{A}^{W}$, we obtain $D(x)=\left(t_{0}+t_{1}\right)^{2}$. To find $B(x)$ we need to find the coefficient of $\tau(1)$ and of $\tau(1) s_{1}$ in $T_{0} T_{1}+T_{1} T_{0}$. The only contribution comes from the terms with $\tau(1)=s_{1} s_{0}$, so from $T_{1} T_{0}$, and we find

$$
B(x)=c_{0}(-x) c_{1}(x) .
$$


To find $C(x)$ we need to find the coefficients of $\tau(-1)$ and $\tau(-1) s_{1}$ in $T_{0} T_{1}+T_{1} T_{0}$. Then we obtain

$$
C(x)=c_{0}(x)\left(2 t_{1}-c_{1}(x)\right)=c_{0}(x) c_{1}(-x) .
$$

(b) Using the duality property for the symmetric Wilson polynomials we find, from (a),

$$
\begin{aligned}
\left(\left(x_{2 m}^{2}-\left(t_{1}+u_{1}\right)^{2}\right) E^{+}\left(x_{2 m}, \gamma_{2 n}\right)=\right. & A^{\sigma}\left(\gamma_{2 n}\right)\left(E^{+}\left(x_{2 m}, \gamma_{2 n}+1\right)-E^{+}\left(x_{2 m}, \gamma_{2 n}\right)\right) \\
& +A^{\sigma}\left(-\gamma_{2 n}\right)\left(E^{+}\left(x_{2 m}, \gamma_{2 n}-1\right)-E^{+}\left(x_{2 m}, \gamma_{2 n}\right)\right) .
\end{aligned}
$$

This identity holds for all $m \in \mathbb{Z}_{\geqslant 0}$, so it holds as an identity in $\mathcal{A}$. Setting $B_{n}=$ $A^{\sigma}\left(\gamma_{2 n}\right), C_{n}=A^{\sigma}\left(-\gamma_{2 n}\right)$, and using $\gamma_{2 n} \pm 1=\gamma_{2 n \pm 2}$, gives the desired relation.

Proposition 3.18 gives precisely the difference equation and recurrence relation for the well-known Wilson polynomials [24]. This gives us the following expression.

Theorem 3.19. The symmetric Wilson polynomials have the explicit expression

$$
E^{+}\left(x, \gamma_{2 n}\right)={ }_{4} F_{3}\left(\begin{array}{c}
-n, n+a+b+c+d-1, a+x, a-x \\
a+b, a+c, a+d
\end{array} ; 1\right) .
$$

Here we use the standard notation for hypergeometric series,

$$
{ }_{p} F_{q}\left(\begin{array}{c}
\alpha_{1}, \ldots, \alpha_{p} \\
\beta_{1}, \ldots, \beta_{q}
\end{array} ; x\right)=\sum_{j=0}^{\infty} \frac{\left(\alpha_{1}\right)_{j} \cdots\left(\alpha_{p}\right)_{j}}{\left(\beta_{1}\right)_{j} \cdots\left(\beta_{q}\right)_{j}} \frac{x^{j}}{j !} .
$$

Using $E^{+}\left(x, \gamma_{2 n}\right)=P_{2 n}^{+}(x) / P_{2 n}^{+}\left(x_{0}\right)$ and the evaluation of $P_{2 n}^{+}\left(x_{0}\right)$ from Proposition 3.14, we now also have an explicit expression for $P_{2 n}^{+}(x)$. Then from Lemma 3.6 and Theorem 3.10 we find an explicit expression for the nonsymmetric Wilson polynomial $p_{m}$ as a sum of two balanced ${ }_{4} F_{3}$-series.

\section{The polynomial Fourier transform}

Let $V$ and $\widetilde{V}$ be an $\mathcal{H}$-module and an $\mathcal{H}_{\sigma}$-module, respectively. A Fourier transform associated to the duality isomorphism $\sigma$ is a linear map $\mathbb{F}: V \rightarrow \widetilde{V}$ that intertwines the actions of $\mathcal{H}$ and $\mathcal{H}_{\sigma}$, i.e.,

$$
\mathbb{F} \circ X=\sigma(X) \circ \mathbb{F} .
$$

We are interested in Fourier transforms that can be written as integral transforms with some kernel. In this section we consider a Fourier transform with the nonsymmetric Wilson polynomials as a kernel. In the next section we consider a Fourier transform with a nonpolynomial kernel.

\subsection{The Fourier transform $\mathbb{F}$}

Let $\Gamma$ denote the spectrum of $-Y \in \mathcal{H}$, i.e., $\Gamma=\left\{-\gamma_{m} \mid m \in \mathbb{Z}_{\geqslant 0}\right\}$, and let $F$ be the space of complex functions on $\Gamma$ with finite support,

$$
F=\{f: \Gamma \rightarrow \mathbb{C} \mid \operatorname{supp}(f) \text { finite }\} .
$$


We define the nonsymmetric polynomial Fourier transform $\mathbb{F}=\mathbb{F}_{\mathbf{t}}: \mathcal{A} \rightarrow F$ by

$$
(\mathbb{F} p)(\gamma)=\langle p, E(\cdot,-\gamma ; \mathbf{t})\rangle_{\mathbf{t}}, \quad \gamma \in \Gamma \text {. }
$$

The mapping $\mathbb{F}$ is injective, since the bilinear form $\langle\cdot, \cdot\rangle_{\mathbf{t}}$ is nondegenerate, and, since the polynomials $E\left(\cdot, \gamma_{m} ; \mathbf{t}\right)$ form an orthogonal basis for $\mathcal{A}$ with respect to $\langle\cdot, \cdot\rangle_{\mathbf{t}}, \mathbb{F}$ is also surjective. For any $f \in F$ let us define the values of $f$ at $\gamma_{0}$ by $f\left(\gamma_{0}\right)=f\left(-\gamma_{0}\right)$. Now we define an action of the affine Weyl group $\mathcal{W}$ on $F$ in the same way as on $\mathcal{A}$, i.e.,

$$
\left(s_{0} f\right)(\gamma)=f(1-\gamma), \quad\left(s_{1} f\right)(\gamma)=f(-\gamma), \quad \gamma \in \Gamma .
$$

Proposition 4.1. The applications

$$
\begin{aligned}
\left(T_{i}^{\sigma} f\right)(\gamma) & =t_{i}^{\sigma} f(\gamma)+c_{i}\left(\gamma ; \mathbf{t}^{\sigma}\right)\left(\left(s_{i} f\right)(\gamma)-f(\gamma)\right), \quad i=0,1, \\
(p(z) f)(\gamma) & =p(\gamma) f(\gamma), \quad p \in \mathcal{A},
\end{aligned}
$$

extend uniquely to a representation of $\mathcal{H}_{\sigma}$ on $F$. With this representation, $\mathbb{F}: \mathcal{A} \rightarrow F$ is a Fourier transform associated to $\sigma$.

Proof. Assuming that the intertwining property (4.1) holds for $X_{1}, X_{2} \in \mathcal{H}$ we have, for $p \in \mathcal{A}$,

$$
\mathbb{F}\left(X_{1} X_{2} p\right)=\sigma\left(X_{1}\right)\left(\mathbb{F}\left(X_{2} p\right)\right)=\sigma\left(X_{1}\right) \sigma\left(X_{2}\right)(\mathbb{F} p)=\sigma\left(X_{1} X_{2}\right)(\mathbb{F} p),
$$

since $\sigma$ is an algebra isomorphism. So it is enough to check the intertwining property for $Y, T_{1}, U_{1} \in \mathcal{H}$, since these elements generate $\mathcal{H}$ as an algebra.

Let $p \in \mathcal{A}$. Then, for $\gamma \in \Gamma$,

$$
\mathbb{F}(Y p)(\gamma)=\langle Y p, E(\cdot,-\gamma)\rangle_{\mathbf{t}}=\langle p, Y E(\cdot,-\gamma)\rangle_{\mathbf{t}}=-\gamma(\mathbb{F} p)(\gamma)
$$

So we obtain

$$
\mathbb{F}(Y p)=-z(\mathbb{F} p)=\sigma(Y)(\mathbb{F} p)
$$

For the action of $T_{1}$ we use Lemma 3.17,

$$
\begin{aligned}
\left(\mathbb{F}\left(T_{1} p\right)\right)(\gamma) & =\left\langle T_{1} p, E(\cdot,-\gamma)\right\rangle_{\mathbf{t}} \\
& =t_{1}\langle p, E(\cdot,-\gamma)\rangle_{\mathbf{t}}+c_{1}^{\sigma}(\gamma)\langle p, E(\cdot, \gamma)-E(\cdot,-\gamma)\rangle_{\mathbf{t}} \\
& =t_{1}(\mathbb{F} p)(\gamma)+c_{1}^{\sigma}(\gamma)((\mathbb{F} p)(-\gamma)-(\mathbb{F} p)(\gamma)) \\
& =\left(T_{1}^{\sigma}(\mathbb{F} p)\right)(\gamma)
\end{aligned}
$$

so $\mathbb{F}\left(T_{1} p\right)=\sigma\left(T_{1}\right)(\mathbb{F} p)$. Recall here that $c^{\sigma}\left(-\gamma_{0}\right)=0$ and $E\left(x,-\gamma_{0}\right)=1$.

Finally, for $U_{1}$ we have, by Lemma 3.17,

$$
\begin{aligned}
\left(\mathbb{F}\left(U_{1} p\right)\right)(\gamma) & =\left\langle U_{1} p, E(\cdot,-\gamma)\right\rangle_{\mathbf{t}} \\
& =u_{1}\langle p, E(\cdot,-\gamma)\rangle_{\mathbf{t}}+c_{0}^{\sigma}(\gamma)\left\langle p_{2}, E(\cdot,-1+\gamma)-E(\cdot,-\gamma)\right\rangle_{\mathbf{t}} \\
& =u_{1}(\mathbb{F} p)(\gamma)+c_{0}^{\sigma}(\gamma)((\mathbb{F} p)(1-\gamma)-(\mathbb{F} p)(\gamma)) \\
& =\left(T_{0}^{\sigma}(\mathbb{F} p)\right)(\gamma),
\end{aligned}
$$

which is the same as $\mathbb{F}\left(U_{1} p\right)=\sigma\left(U_{1}\right)(\mathbb{F} p)$. 


\subsection{The inverse transform}

We define a weight function $w$ on $\Gamma$ by

$$
w(\gamma ; \mathbf{t})=\operatorname{Res}_{y=\gamma} \Delta\left(y ; \mathbf{t}^{\sigma}\right) .
$$

Let us associate parameters $\tilde{a}, \tilde{b}, \tilde{c}, \tilde{d}$ to the multiplicity function $\mathbf{t}^{\sigma}$ in the same way as (3.1), i.e.,

$$
(\tilde{a}, \tilde{b}, \tilde{c}, \tilde{d})=\left(t_{1}+t_{0}, t_{1}-t_{0}, u_{1}+u_{0}+\frac{1}{2}, u_{1}-u_{0}+\frac{1}{2}\right) .
$$

In view of the explicit expression for $\Delta, \operatorname{Res}_{y=-n} \Gamma(y)=(-1)^{n} / n !, n \in \mathbb{Z}_{\geqslant 0}$, and $\Gamma(n+1)=n !$, we have

$$
\begin{aligned}
& w(\gamma ; \mathbf{t}) \\
& = \begin{cases}(-1)^{n-1} \frac{\Gamma(\tilde{a}+\gamma) \Gamma(\tilde{b}+\gamma) \Gamma(\tilde{b}+1-\gamma) \Gamma(\tilde{c} \pm \gamma) \Gamma(\tilde{d} \pm \gamma)}{\Gamma(\gamma-\tilde{a}) \Gamma(2 \gamma) \Gamma(1-2 \gamma)}, & \gamma=\tilde{a}+n, \quad n \in \mathbb{N}, \\
(-1)^{n} \frac{\Gamma(\tilde{a}+1-\gamma) \Gamma(\tilde{b}+\gamma) \Gamma(\tilde{b}+1-\gamma) \Gamma(\tilde{c} \pm \gamma) \Gamma(\tilde{d} \pm \gamma)}{\Gamma(1-\gamma-\tilde{a}) \Gamma(2 \gamma) \Gamma(1-2 \gamma)}, & \gamma=-(\tilde{a}+n), \quad n \in \mathbb{Z}_{\geqslant 0} .\end{cases}
\end{aligned}
$$

To this weight function we associate a bilinear form $[\cdot, \cdot]_{\mathbf{t}}: F \times F \rightarrow \mathbb{C}$ by

$$
[f, g]_{\mathbf{t}}=\sum_{\gamma \in \Gamma} f(\gamma) g(-\gamma) w(\gamma ; \mathbf{t})
$$

Now we define the map $\mathbb{G}=\mathbb{G}_{\mathbf{t}}: F \rightarrow \mathcal{A}$ by

$$
(\mathbb{G} f)(x)=[f, E(x, ; ; \mathbf{t})]_{\mathbf{t}}, \quad f \in F, \quad x \in \mathbb{C} .
$$

Note here that $E(x, \cdot) \notin F$, but clearly for any $f \in F$ the function $\mathbb{G} f$ exists.

Proposition 4.2. For $X \in \mathcal{H}_{\sigma}$ and $f \in F$ we have

$$
\mathbb{G}(X f)=\sigma^{-1}(X)(\mathbb{G} f) .
$$

Proof. Let $f \in F$. It is enough to check the statement for $X=z, T_{0}^{\sigma}, T_{1}^{\sigma}$. For $X=z$ we obtain

$$
\begin{aligned}
\mathbb{G}(z f)(x) & =\sum_{\gamma \in \Gamma} \gamma f(\gamma) E(x,-\gamma) w(\gamma) \\
& =\sum_{\gamma \in \Gamma} f(\gamma)(-Y E(\cdot,-\gamma))(x) w(\gamma) \\
& =(-Y(\mathbb{G} f))(x) \\
& =\left(\sigma^{-1}(z)(\mathbb{G} f)\right)(\gamma) .
\end{aligned}
$$


Next, for $X=T_{0}^{\sigma}$ we obtain

$$
\begin{aligned}
\mathbb{G}\left(T_{0}^{\sigma} f\right)(x)= & \sum_{\gamma \in \Gamma}\left(u_{1} f(\gamma)+\frac{\left(u_{1}+u_{0}+\frac{1}{2}-\gamma\right)\left(u_{1}-u_{0}+\frac{1}{2}-\gamma\right)}{1-2 \gamma}(f(1-\gamma)-f(\gamma))\right) \\
& \times E(x,-\gamma) w(\gamma) \\
= & \sum_{\gamma \in \Gamma} f(\gamma)\left(u_{1} E(x,-\gamma)+\frac{(\tilde{c}-\gamma)(\tilde{d}-\gamma)}{1-2 \gamma}(E(x, \gamma-1)-E(x,-\gamma))\right) w(\gamma) \\
= & \sum_{\gamma \in \Gamma} f(\gamma)\left(U_{0} E(\cdot ;-\gamma)\right)(x) w(\gamma) \\
= & \left(\sigma^{-1}\left(T_{0}^{\sigma}\right)(\mathbb{G} f)\right)(x) .
\end{aligned}
$$

Here we use Lemma 3.17, and

$$
\frac{(\tilde{c}-\gamma)(\tilde{d}-\gamma)}{1-2 \gamma} w(\gamma ; \mathbf{t})=\frac{(\tilde{c}-1+\gamma)(\tilde{d}-1+\gamma)}{2 \gamma-1} w(1-\gamma ; \mathbf{t}),
$$

which can be obtained from the explicit expression for $w(\gamma ; \mathbf{t})$.

Finally, using Lemma 3.17 we find, for $T_{1}^{\sigma}$,

$$
\begin{aligned}
\mathbb{G}\left(T_{1}^{\sigma} f\right)(x) & =\sum_{\gamma \in \Gamma}\left(t_{1} f(\gamma)+\frac{\left(t_{1}+t_{0}+\gamma\right)\left(t_{1}-t_{0}+\gamma\right)}{2 \gamma}(f(-\gamma)-f(\gamma))\right) E(x,-\gamma) w(\gamma) \\
& =\sum_{\gamma \in \Gamma} f(\gamma)\left(t_{1} E(x,-\gamma)+\frac{(\tilde{a}+\gamma)(\tilde{b}+\gamma)}{2 \gamma}(E(x, \gamma)-E(x,-\gamma))\right) w(\gamma) \\
& =\sum_{\gamma \in \Gamma} f(\gamma)\left(T_{1} E(\cdot,-\gamma)(x) w(\gamma)\right. \\
& =\left(\sigma^{-1}\left(T_{1}^{\sigma}\right)(\mathbb{G} f)\right)(x),
\end{aligned}
$$

where we have used

$$
\frac{(\tilde{a}+\gamma)(\tilde{b}+\gamma)}{2 \gamma} w(\gamma ; \mathbf{t})=\frac{(\tilde{a}-\gamma)(\tilde{b}-\gamma)}{-2 \gamma} w(-\gamma ; \mathbf{t})
$$

for $\gamma \neq-\gamma_{0}$. This proves the proposition.

From combining Propositions 4.1 and 4.2 it follows that $\mathbb{G}$ is, up to a constant, the inverse of $\mathbb{F}$. This enables us to determine the "quadratic norms" for the nonsymmetric Wilson polynomials.

\section{Theorem 4.3.}

(a) We have $\mathbb{G} \circ \mathbb{F}=\mathcal{N} i d_{\mathcal{A}}$, and $\mathbb{F} \circ \mathbb{G}=\mathcal{N} i d_{F}$, where $\mathcal{N}=\mathcal{N}(\mathbf{t})=\langle 1,1\rangle_{\mathbf{t}} w\left(-\gamma_{0} ; \mathbf{t}\right)$.

(b) For $\gamma \in-\Gamma$ we have

$$
\frac{\langle E(\cdot, \gamma ; \mathbf{t}), E(\cdot, \gamma ; \mathbf{t})\rangle_{\mathbf{t}}}{\langle 1,1\rangle_{\mathbf{t}}}=\frac{w\left(-\gamma_{0} ; \mathbf{t}\right)}{w(-\gamma ; \mathbf{t})} .
$$


Proof. (a) Let $p \in \mathcal{A}$. From Propositions 4.1 and 4.2 we obtain

$$
\mathbb{G}(\mathbb{F} p)=\mathbb{G}(\sigma(p(z))(\mathbb{F} 1))=p(z)(\mathbb{G}(\mathbb{F} 1)) .
$$

Using the orthogonality relations for the nonsymmetric Wilson polynomials, and the definitions of $\mathbb{F}$ and $\mathbb{G}$, we obtain

$$
\mathbb{G}(\mathbb{F} 1)=\langle 1,1\rangle_{\mathbf{t}} w\left(-\gamma_{0} ; \mathbf{t}\right) .
$$

So we have $\mathbb{G} \circ \mathbb{F}=\mathcal{N} i d_{\mathcal{A}}$ with $\mathcal{N}$ as in the theorem.

Next let $f \in F$. Since $\mathbb{F}$ is bijective, we have $f=\mathbb{F} p$ for some $p \in \mathcal{A}$. This gives us

$$
\mathbb{F}(\mathbb{G} f)=(\mathbb{F} \mathbb{G})(\mathbb{F} p)=\mathcal{N}(\mathbb{F} p)=\mathcal{N} f,
$$

which shows that $\mathbb{F} \circ \mathbb{G}=\mathcal{N} i d_{F}$.

(b) Using the orthogonality relations for the nonsymmetric Wilson polynomials, and the definitions of $\mathbb{F}$ and $\mathbb{G}$, we find

$$
(\mathbb{G}(\mathbb{F} E(\cdot,-\gamma)))(x)=\langle E(\cdot,-\gamma), E(\cdot,-\gamma)\rangle_{\mathbf{t}} E(x ;-\gamma) w(\gamma ; \mathbf{t}), \quad \gamma \in \Gamma .
$$

On the other hand, from (a) we obtain $(\mathbb{G}(\mathbb{F} E(\cdot,-\gamma)))(x)=\mathcal{N} E(x,-\gamma)$. Comparing gives

$$
\langle E(\cdot,-\gamma), E(\cdot,-\gamma)\rangle_{\mathbf{t}}=\frac{\mathcal{N}}{w(\gamma ; \mathbf{t})},
$$

which is equivalent to statement (b) in the theorem.

Let us remark that since $\langle f, g\rangle_{\mathbf{t}}=\frac{1}{2}(a+b)\langle f, g\rangle_{\mathbf{t}}^{+}$for $f, g \in \mathcal{A}^{W}$, and $\langle 1,1\rangle_{\mathbf{t}}^{+}$is a well-known integral (see [24]), we can evaluate $\langle 1,1\rangle_{\mathbf{t}}$;

$$
\langle 1,1\rangle_{\mathbf{t}}=\frac{\Gamma(a+b+1) \Gamma(a+c) \Gamma(a+d) \Gamma(b+c) \Gamma(b+d) \Gamma(c+d)}{\Gamma(a+b+c+d)} .
$$

So the "quadratic norms" of the nonsymmetric Wilson polynomials are completely explicit.

For $\mathbb{F}$ and $\mathbb{G}$ we have the following Plancherel-type formulas.

Proposition 4.4. For $p_{1}, p_{2} \in \mathcal{A}$ and $f_{1}, f_{2} \in F$,

$$
\left[\mathbb{F} p_{1}, \mathbb{F} p_{2}\right]_{\mathbf{t}}=\mathcal{N}\left\langle p_{1}, p_{2}\right\rangle_{\mathbf{t}}, \quad\left\langle\mathbb{G} f_{1}, \mathbb{G} f_{2}\right\rangle_{\mathbf{t}}=\mathcal{N}\left[f_{1}, f_{2}\right]_{\mathbf{t}}
$$

Proof. From the definitions of $\mathbb{F}$ and $\mathbb{G}$ we obtain, for $p \in \mathcal{A}$ and $f \in F$,

$$
\begin{aligned}
{[f, \mathbb{F} p]_{\mathbf{t}} } & =\sum_{\gamma \in \Gamma} f(\gamma)\left(\frac{1}{2 \pi i} \int_{\mathcal{C}} p(x) E(x,-\gamma) \Delta(x) d x\right) w(\gamma) \\
& =\frac{1}{2 \pi i} \int_{\mathcal{C}}\left(\sum_{\gamma \in \Gamma} f(\gamma) E(x,-\gamma) w(\gamma)\right) p(x) \Delta(x) d x \\
& =\langle\mathbb{G} f, p\rangle_{\mathbf{t}} .
\end{aligned}
$$

For the first identity take $f=\mathbb{F} p_{1}$ and $p=p_{2}$ in (4.3), for the second identity take $f=f_{1}$ and $p=\mathbb{G} f_{2}$. Then use Theorem 4.3. 


\subsection{The symmetric transform}

The nonsymmetric polynomial Fourier transform is defined as an integral transform with the nonsymmetric Wilson polynomial as a kernel. Similarly we can define a symmetric polynomial Fourier transform as an integral transform with the symmetric Wilson polynomial as a kernel. For this transform we can also find the inverse, and obtain Plancherel-type formulas. The proofs for these are very similar to the proofs for the nonsymmetric case, and will be left to the reader.

Let $\Gamma^{+}=\left\{\gamma_{2 n} \mid n \in \mathbb{Z}_{\geqslant 0}\right\} \subset-\Gamma$. Furthermore, let $F_{+}$denote the subspace of $F$ consisting of $W$-invariant functions on $\Gamma$, then

$$
F_{+}=\left\{f \in F \mid f(\gamma)=f(-\gamma), \gamma \in \Gamma^{+}\right\} .
$$

We define the symmetric polynomial Fourier transform $\mathbb{F}^{+}: \mathcal{A}_{+} \rightarrow F_{+}$by

$$
\left(\mathbb{F}^{+} p\right)(\gamma)=\left\langle p, E^{+}(\cdot, \gamma)\right\rangle_{\mathbf{t}}^{+}, \quad \gamma \in \Gamma^{+}, \quad p \in \mathcal{A}_{+} .
$$

Let $w^{+}$be the weight function on $\Gamma^{+}$defined by

$$
w^{+}(\gamma ; \mathbf{t})=\operatorname{Res}_{y=\gamma} \Delta^{+}\left(y ; \mathbf{t}^{\sigma}\right)
$$

With this weight function we define a bilinear form $[\cdot, \cdot]_{\mathbf{t}}^{+}: F_{+} \times F_{+} \rightarrow \mathbb{C}$ by

$$
[f, g]_{\mathbf{t}}^{+}=\sum_{\gamma \in \Gamma^{+}} f(\gamma) g(\gamma) w^{+}(\gamma ; \mathbf{t})
$$

Now we define $\mathbb{G}^{+}: F_{+} \rightarrow \mathcal{A}_{+}$by

$$
\left(\mathbb{G}^{+} f\right)(x)=\left[f, E^{+}(x, \cdot)\right]_{\mathbf{t}}^{+}, \quad x \in \mathbb{C}, \quad f \in F_{+} .
$$

In the same way as in the previous section we can show that $\mathbb{G}^{+}$is, up to a multiplicative constant, the inverse of $\mathbb{F}^{+}$, and this leads to the (well-known) evaluation of the quadratic norms of the symmetric Wilson polynomials and Plancherel-type formulas.

\section{Theorem 4.5.}

(a) We have $\mathbb{G}^{+} \circ \mathbb{F}^{+}=\mathcal{N}^{+} i d_{\mathcal{A}_{+}}$, and $\mathbb{F}^{+} \circ \mathbb{G}^{+}=\mathcal{N}^{+} i d_{F_{+}}$, where $\mathcal{N}^{+}=\mathcal{N}^{+}(\mathbf{t})=$ $\langle 1,1\rangle_{\mathbf{t}}^{+} w^{+}\left(\gamma_{0} ; \mathbf{t}\right)$.

(b) For $\gamma \in \Gamma^{+}$,

$$
\frac{\left\langle E^{+}(\cdot, \gamma ; \mathbf{t}), E^{+}(\cdot, \gamma ; \mathbf{t})\right\rangle_{\mathbf{t}}^{+}}{\langle 1,1\rangle_{\mathbf{t}}^{+}}=\frac{w^{+}\left(\gamma_{0} ; \mathbf{t}\right)}{w^{+}(\gamma ; \mathbf{t})} .
$$

(c) For $p_{1}, p_{2} \in \mathcal{A}_{+}$and $f_{1}, f_{2} \in F_{+}$,

$$
\left[\mathbb{F}^{+} p_{1}, \mathbb{F}^{+} p_{2}\right]_{\mathbf{t}}^{+}=\mathcal{N}^{+}\left\langle p_{1}, p_{2}\right\rangle_{\mathbf{t}}^{+}, \quad\left\langle\mathbb{G}^{+} f_{1}, \mathbb{G}^{+} f_{2}\right\rangle_{\mathbf{t}}^{+}=\mathcal{N}^{+}\left[f_{1}, f_{2}\right]_{\mathbf{t}}^{+}
$$




\section{The nonpolynomial Fourier transform}

In this section we study a Fourier transform associated to $\sigma$ with a nonpolynomial kernel. This kernel turns out to be a meromorphic continuation of the nonsymmetric Wilson polynomial in its degree.

\subsection{The Gaussian}

We define an involution $\tau$ on multiplicity functions by interchanging the values on the $a_{0}$-orbit and the $a_{0}^{\vee}$-orbit; given $\mathbf{t}=\left(t_{0}, u_{0}, t_{1}, u_{1}\right)$ we have

$$
\mathbf{t}^{\tau}=\left(u_{0}, t_{0}, t_{1}, u_{1}\right) \text {. }
$$

We use notations with $\tau$ in the same way we do with the involution $\sigma$. We will also compose $\sigma$ and $\tau$, and we use notations like $\mathbf{t}^{\sigma \tau}=\left(\mathbf{t}^{\sigma}\right)^{\tau}=\left(u_{0}, u_{1}, t_{1}, t_{0}\right)$. We will use similar notations for objects depending on $\mathbf{t}^{\sigma \tau}$, e.g., $H_{\sigma \tau}=H\left(\mathbf{t}^{\sigma \tau}\right)$. We mention that

$$
\mathbf{t}^{\tau \sigma \tau}=\left(t_{0}, u_{1}, t_{1}, u_{0}\right)=\mathbf{t}^{\sigma \tau \sigma},
$$

which will be used later on.

We define the Gaussian by

$$
G(x ; \mathbf{t})=\Gamma\left(t_{0}-u_{0}+\frac{1}{2} \pm x\right) .
$$

This function will play the same role as $e^{-x^{2}}$ does for the Hankel transform, see [5]. Let $\tau$ denote conjugation with the Gaussian;

$$
G \circ X=\tau(X) \circ G, \quad X \in \mathcal{H} .
$$

Proposition 5.1. The application $X \mapsto \tau(X)$ is an algebra isomorphism $\mathcal{H} \rightarrow \mathcal{H}_{\tau}$. On the generators $z, T_{0}, T_{1}$ of $\mathcal{H}$ the action of $\tau$ is given by

$$
\tau(z)=z, \quad \tau\left(T_{0}\right)=U_{0}^{\tau}, \quad \tau\left(T_{1}\right)=T_{1}^{\tau} .
$$

Proof. The fact that $\tau$ is an algebra isomorphism follows from the explicit action on the generators, so we need to check the actions given in the lemma.

The identities $G z G^{-1}=z$ and $G T_{1} G^{-1}=T_{1}^{\tau}$ are easy to check, using that $z$ and $T_{1}$ do not depend on $t_{0}$ and $u_{0}$, and the identity $\left(s_{1} G^{-1}\right)(x)=G(x)^{-1}$. For $T_{0}$ we use

$$
\left(s_{0} G^{-1}\right)(x)=G(1-x)^{-1}=\frac{t_{0}-u_{0}-\frac{1}{2}+x}{t_{0}-u_{0}+\frac{1}{2}-x} G(x)^{-1},
$$

then we obtain, for $p \in \mathcal{A}$,

$$
\left(\tau\left(T_{0}\right) p\right)(x)=\left(t_{0}-c_{0}(x)\right) p(x)+\frac{\left(t_{0}+u_{0}+\frac{1}{2}-x\right)\left(t_{0}-u_{0}-\frac{1}{2}+x\right)}{1-2 x} p(1-x) .
$$

By a direct calculation it is verified that

$$
t_{0}-c_{0}(x)=x-\frac{1}{2}-u_{0}+c_{0}^{\tau}(x) .
$$

So we find

$$
\left(\tau\left(T_{0}\right) p\right)(x)=\left(x-\frac{1}{2}-u_{0}\right) p(x)-c_{0}^{\tau}(x)(p(1-x)-p(x))=\left(\left(z-\frac{1}{2}-T_{0}^{\tau}\right) p\right)(x),
$$

which gives $\tau\left(T_{0}\right)=U_{0}^{\tau}$. 


\subsection{Construction of a Fourier transform}

We construct a Fourier transform associated to $\sigma$ using the transforms $\mathbb{F}, \mathbb{G}$ from the previous section, and the Gaussian. First, we define a linear operator $\mathfrak{F}: \mathcal{A} \rightarrow \mathcal{A}$ by

$$
\mathfrak{F}=\mathbb{G}_{\sigma \tau} \circ G_{\tau \sigma} \circ \mathbb{F}_{\tau} .
$$

Note that the set $\Gamma_{\tau}$ consists of the points

$$
-\gamma_{m}^{\tau}= \begin{cases}-\left(u_{0}+t_{1}+n\right), & m=2 n, \\ u_{0}+t_{1}+n, & m=2 n-1,\end{cases}
$$

which are clearly invariant under $u_{1} \leftrightarrow t_{0}$. So we have $\Gamma_{\tau}=\Gamma_{\sigma \tau}$. Recall that $F$ is the set of finitely supported functions on $\Gamma$, then it follows that $F^{\tau}=F^{\sigma \tau}$. So the composition $\mathbb{G}_{\sigma \tau} \circ G_{\tau \sigma} \circ \mathbb{F}_{\tau}$ is well defined.

Proposition 5.2. For $X \in \mathcal{H}_{\tau}$ we have

$$
\mathfrak{F} \circ X=\chi(X) \circ \mathfrak{F}
$$

where $\chi: \mathcal{H}_{\tau} \rightarrow \mathcal{H}_{\sigma \tau}$ is the isomorphism $\chi=\sigma_{\sigma \tau}^{-1} \circ \tau_{\tau \sigma} \circ \sigma_{\tau}$. Explicitly, $\chi: \mathcal{H}_{\tau} \rightarrow \mathcal{H}_{\sigma \tau}$ is given on algebraic generators of $\mathcal{H}_{\tau}$ by

$$
\chi(z)=-U_{0}^{\sigma \tau}-T_{1}^{\sigma \tau}, \quad \chi\left(Y^{\tau}\right)=Y^{\sigma \tau}, \quad \chi\left(T_{1}^{\tau}\right)=T_{1}^{\sigma \tau} .
$$

Proof. First we check the intertwining property. Let $X \in \mathcal{H}_{\tau}$ and $p \in \mathcal{A}$. Using the intertwining properties of $\mathbb{F}$ and $G$, see Proposition 4.1 and (5.1), we have

$$
\begin{aligned}
\mathfrak{F}(X p) & =\left(\mathbb{G}_{\sigma \tau} \circ G_{\tau \sigma} \circ \mathbb{F}_{\tau}\right)(X p) \\
& =\left(\mathbb{G}_{\sigma \tau} \circ G_{\tau \sigma}\right)\left(\sigma_{\tau}(X)\left(\mathbb{F}_{\tau} p\right)\right) \\
& =\mathbb{G}_{\sigma \tau}\left[\left(\tau_{\tau \sigma} \circ \sigma_{\tau}\right)(X)\left(\left(G_{\tau \sigma} \circ \mathbb{F}_{\tau}\right)(p)\right)\right] .
\end{aligned}
$$

Note that $\left(\tau_{\tau \sigma} \circ \sigma_{\tau}\right)(X) \in \mathcal{H}_{\tau \sigma \tau}=\mathcal{H}_{\sigma \tau \sigma}$. So by the intertwining property of $\mathbb{G}$, see Proposition 4.2, it follows that

$$
\mathfrak{F}(X p)=\left(\sigma_{\sigma \tau}^{-1} \circ \tau_{\tau \sigma} \circ \sigma_{\tau}\right)(X)\left(\left(\mathbb{G}_{\sigma \tau} \circ G_{\tau \sigma} \circ \mathbb{F}_{\tau}\right)(p)\right)=\chi(X)(\mathfrak{F} p) .
$$

The explicit expressions for $\chi(X), X=z, Y^{\tau}, T_{1}^{\tau} \in \mathcal{H}_{\tau}$, are easily verified using the explicit actions of $\sigma$ and $\tau$ on algebraic generators.

We define the symmetric version $\mathfrak{F}^{+}: \mathcal{A}_{+} \rightarrow \mathcal{A}_{+}$of the operator $\mathfrak{F}$ by

$$
\mathfrak{F}^{+}=\mathbb{G}_{\sigma \tau}^{+} \circ G_{\tau \sigma} \circ \mathbb{F}_{\tau}^{+} .
$$

\section{Proposition 5.3.}

(a) Let $\mathfrak{L}: \mathcal{A} \rightarrow \mathcal{A}$ be the linear operator defined on the Wilson polynomials by

$$
\left(\mathfrak{L} E_{\tau}(\cdot, \gamma)\right)(\lambda)=G_{\tau \sigma \tau}(\gamma) E_{\sigma \tau}(\lambda, \gamma), \quad \gamma \in-\Gamma_{\tau},
$$

then $\mathfrak{L}=\mathcal{N}_{\tau}^{-1} \mathfrak{F}$, where $\mathcal{N}$ denotes the constant from Theorem 4.3.

(b) Let $\mathfrak{L}^{+}: \mathcal{A}_{+} \rightarrow \mathcal{A}_{+}$be the linear operator defined on the symmetric Wilson polynomials by

$$
\left(\mathfrak{L}^{+} E_{\tau}^{+}(\cdot, \gamma)\right)(\lambda)=G_{\tau \sigma \tau}(\gamma) E_{\sigma \tau}^{+}(\lambda, \gamma), \quad \gamma \in \Gamma_{\tau}^{+},
$$

then $\mathfrak{L}^{+}=\left(\mathcal{N}_{\tau}^{+}\right)^{-1} \mathfrak{F}^{+}$, where $\mathcal{N}^{+}$denotes the constant from Theorem 4.5. 
Proof. (a) Let $\mathcal{L}$ and $\mathcal{L}^{\prime}$ be linear operators on $\mathcal{A}$, such that $\mathfrak{L} E_{\tau}(\cdot, \gamma)=\mathfrak{L}^{\prime} E_{\tau}(\cdot, \gamma)$. The Wilson polynomials $E_{\tau}(\cdot, \gamma), \gamma \in-\Gamma_{\tau}$, form a linear basis for $\mathcal{A}$, therefore $\mathfrak{L}=\mathfrak{L}^{\prime}$. So, to prove the proposition, we need to calculate the action of $\mathfrak{F}$ on the Wilson polynomials $E_{\tau}$.

Let $\gamma, \gamma^{\prime} \in \Gamma_{\tau}$. By Theorem 4.3(b) we have

$$
\left(\mathbb{F}_{\tau} E_{\tau}(\cdot,-\gamma)\right)\left(\gamma^{\prime}\right)=\left\langle E_{\tau}(\cdot,-\gamma), E_{\tau}\left(\cdot,-\gamma^{\prime}\right)\right\rangle_{\mathbf{t}^{\tau}}=\delta_{\gamma, \gamma^{\prime}} \frac{\mathcal{N}_{\tau}}{w_{\tau}(\gamma)}
$$

Multiplying this expression by $G_{\tau \sigma}\left(\gamma^{\prime}\right)$ and applying $\mathbb{G}_{\sigma \tau}$ then leads to

$$
\left(\mathfrak{F} E_{\tau}(\cdot,-\gamma)\right)(\lambda)=\mathcal{N}_{\tau} G_{\tau \sigma}(\gamma) \frac{w_{\sigma \tau}(\gamma)}{w_{\tau}(\gamma)} E_{\sigma \tau}(\lambda,-\gamma)
$$

Using the definition of the weight $\Delta$ we see that $G_{\tau} \Delta=G \Delta_{\tau}$, so that by the definition of the weight $w$ and by $\mathbf{t}^{\tau \sigma}=\mathbf{t}^{\sigma \tau \sigma \tau}$ it follows that

$$
G_{\tau \sigma}(\gamma) w_{\sigma \tau}(\gamma)=G_{\tau \sigma \tau}(\gamma) w_{\tau}(\gamma), \quad \gamma \in \Gamma_{\tau}
$$

So we find that $\mathfrak{F} E_{\tau}(\cdot,-\gamma)=\mathcal{N}_{\tau} \mathfrak{L} E_{\tau}(\cdot,-\gamma)$.

(b) This is proved in the same way as (a) using the orthogonality relations for the symmetric Wilson polynomials.

Now define the operator $\mathcal{F}^{\prime}: \mathcal{A} G_{\tau} \rightarrow \mathcal{A} G_{\sigma \tau}$ by

$$
\mathcal{F}^{\prime}=G_{\sigma \tau} \circ \mathfrak{F} \circ G_{\tau}^{-1}
$$

Proposition 5.4. The operator $\mathcal{F}^{\prime}$ is a Fourier transform associated to $\sigma$.

Proof. We need to show that $\mathcal{F}^{\prime} \circ X=\sigma(X) \circ \mathcal{F}^{\prime}$ for all $X \in \mathcal{H}$. Let $p \in \mathcal{A}$ and let $f=p G_{\tau}$. From (5.1) and Proposition 5.2(a) we obtain

$$
\begin{aligned}
\mathcal{F}^{\prime}(X f) & =G_{\sigma \tau} \mathfrak{F}^{\prime}(\tau(X) p)=G_{\sigma \tau}[(\chi \circ \tau)(X)](\mathfrak{F} p) \\
& =\left[\left(\tau_{\sigma \tau} \circ \chi \circ \tau\right)(X)\right]\left(G_{\sigma \tau} \mathfrak{F} p\right)=\left[\left(\tau_{\sigma \tau} \circ \chi \circ \tau\right)(X)\right]\left(\mathcal{F}^{\prime} f\right) .
\end{aligned}
$$

Now we must check that $\sigma=\tau_{\sigma \tau} \circ \chi \circ \tau$. It is enough to check that this identity is true on generators of $\mathcal{H}$. This is a straightforward exercise that we leave to the reader.

\subsection{The Fourier transform $\mathcal{F}$}

We want to write $\mathcal{F}^{\prime}$ as an integral transform with some kernel. First we consider the related transform $\mathfrak{F}$. Writing out explicitly $\mathfrak{F} p=\left(\mathbb{G}_{\sigma \tau} \circ G_{\tau \sigma} \circ \mathbb{F}_{\tau}\right) p$ and changing the order of summation and integration, suggests that we may write

$$
(\mathfrak{F} p)(\lambda)=k\langle p, \mathfrak{E}(\cdot, \lambda)\rangle_{\mathbf{t}^{\tau}}, \quad p \in \mathcal{A},
$$

for some kernel $\mathfrak{E}(x, \lambda)$ and $k$ a nonzero constant. 
Remark 5.5. If we formally expand the kernel $\mathfrak{E}(x, \lambda)$ in terms of Wilson polynomials $E_{\tau}(x, \gamma)$ and we calculate the coefficients of $E_{\tau}(x, \gamma)$ using the orthogonality relations for the Wilson polynomials, we obtain

$$
\mathfrak{E}(x, \lambda)=k^{-1} \sum_{\gamma \in \Gamma_{\tau}} E_{\tau}(x ;-\gamma) E_{\sigma \tau}(\lambda ;-\gamma) G_{\tau \sigma}(\gamma) w_{\sigma \tau}(\gamma)
$$

This gives a formal power series for the kernel $\mathfrak{E}(x, \lambda)$ similar to the kernels defined by Cherednik $[3,(5.12)]$, see also [23, (5.13)]. However, it turns out here that this sum does not converge absolutely. In [3], [23] the absolute convergence of the series comes from the Gaussian, which, in the rank 1 case, contains the factor $q^{n^{2}}$ for $0<q<1$, where $n$ is the summation index. Although we cannot define the kernel in this way, it does give us an idea what properties the kernel $\mathfrak{E}$ is expected to have; if the above expansion would converge absolutely, $\mathfrak{E}(x, \lambda)$ would be an entire function in $x$ and $\lambda$, and $\mathfrak{E}$ would satisfy the duality property $\mathfrak{E}(x, \lambda)=\mathfrak{E}_{\sigma}(\lambda, x)$, provided that $k_{\sigma}=k$.

We want to find a kernel $\mathfrak{E}$ such that the integral transform (5.4) maps a Wilson polynomial $E_{\tau}(\cdot, \gamma)$ to a multiple of $G_{\tau \sigma \tau}(\gamma) E_{\sigma \tau}(\cdot, \gamma)$ as in Proposition 5.3(a). To do this we introduce the function

$$
\begin{aligned}
\phi_{\lambda}(x ; \mathbf{t})= & \frac{\Gamma(1-a-d)}{\Gamma(a+b) \Gamma(a+c) \Gamma(1-d \pm x) \Gamma(1-\tilde{d} \pm \lambda)} \\
& \times{ }_{4} F_{3}\left(\begin{array}{c}
a+x, a-x, \tilde{a}+\lambda, \tilde{a}-\lambda \\
a+b, a+c, a+d
\end{array} ;\right) \\
& +\frac{\Gamma(a+d-1)}{\Gamma(1+b-d) \Gamma(1+c-d) \Gamma(a \pm x) \Gamma(\tilde{a} \pm \lambda)} \\
& \quad \times{ }_{4} F_{3}\left(\begin{array}{c}
1-d+x, 1-d-x, 1-\tilde{d}+\lambda, 1-\tilde{d}-\lambda \\
1+b-d, 1+c-d, 2-a-d
\end{array} ;\right) .
\end{aligned}
$$

In [8] the function $\phi_{\lambda}$ is called a Wilson function. Here we will use the name "Wilson function" for a different (but closely related) function, see Definition 5.10. Using transformation formulas for hypergeometric functions, the function $\phi_{\lambda}$ can be expressed as a multiple of a very-well-poised ${ }_{7} F_{6}$-function. In [8] a second-order difference operator $L$ is studied, which in the notation of this paper can be written as $L=\tilde{a}^{2}-G_{\tau}^{-1} \circ\left(Y^{2}\right)_{\text {sym }} \circ G_{\tau}$. From results of Ismail, Letessier, Valent, and Wimp [9], and Masson [14], who studied the associated Wilson polynomials using contiguous relations for ${ }_{7} F_{6}$-series, it follows that the function $\phi_{\lambda}$ is an eigenfunction of $L$ for eigenvalue $\tilde{a}^{2}-\lambda^{2}$.

The following properties of $\phi_{\lambda}$ will be useful for us.

Lemma 5.6. The function $\phi_{\lambda}(x)$ has the following properties:

(a) $\phi_{\lambda}(x)$ is an entire function in $x$ and $\lambda$.

(b) For $p \in \mathcal{A}$ and $y \in \mathbb{R}$, the integral $\left\langle p, \phi_{\lambda}(\cdot+y)\right\rangle_{\mathbf{t}^{\tau}}^{+}$converges absolutely.

(c) For $\gamma \in \Gamma_{\tau}^{+}$,

$$
\left\langle E_{\tau}^{+}(\cdot, \gamma), \phi_{\lambda}\right\rangle_{\mathbf{t}^{\tau}}^{+}=\frac{2 G_{\tau \sigma \tau}(\gamma)}{G_{\tau \sigma \tau}\left(\gamma_{0}^{\tau}\right)} E_{\sigma \tau}^{+}(\lambda, \gamma)
$$

Note that for (b) the only conditions on the values of $t_{0}, u_{0}, t_{1}, u_{1}$ are the conditions from Subsection 3.1. 
Proof. Property (a) follows directly from the definition of $\phi_{\lambda}(x)$. Using a transformation formula for ${ }_{7} F_{6}$-series, it is proved in [8] that, for $x \rightarrow \pm \infty$ and $y \in \mathbb{R}$,

$$
\phi_{\lambda}(y+i x) \sim|x|^{d-a-b-c} e^{\pi(|x|-i y)}\left(c(\lambda)|x|^{-2 \lambda}+c(-\lambda)|x|^{2 \lambda}\right),
$$

where $c(\lambda)$ is independent of $x$ and $y$. The weight function $\Delta_{\tau}^{+}$has the asymptotic behavior

$$
\Delta_{\tau}^{+}(x) \sim|x|^{2 a+2 b+2 c-2 d-1} e^{-2 \pi|x|}, \quad x \rightarrow \pm i \infty,
$$

which can be obtained from applying Euler's reflection formula for the $\Gamma$-function and using the asymptotic formula

$$
\frac{\Gamma(\alpha+z)}{\Gamma(\beta+z)} \sim z^{\alpha-\beta}, \quad|z| \rightarrow \infty, \quad|\arg (z)|<\pi
$$

Now we see that the integral $\left\langle p, \phi_{\lambda}(\cdot+y)\right\rangle_{\mathbf{t}^{\tau}}^{+}$is absolutely convergent, independent of the values of $a, b, c, d$. Property (c) is Theorem 6.9 in [8], where it is proved in case the parameters $a, b, c, d$ are such that $\Delta_{\tau}^{+}(x)$ is a positive weight on $i \mathbb{R}$, but the proof remains valid without these conditions.

From Lemma 5.6(c) and Proposition 5.3(b) it follows immediately that $\mathfrak{F}^{+}$can be written as an integral transform with $\phi_{\lambda}$ as a kernel. Next we use $\phi_{\lambda}$ to write $\mathfrak{F}$ as an integral transform. Recall the generalized Weyl character formula from Theorem 3.10. For the renormalized Wilson polynomials this formula looks as follows.

Lemma 5.7. For $m \in \mathbb{N}$ the renormalized antisymmetric Wilson polynomial satisfies

$$
E^{-}\left(x, \gamma_{m} ; \mathbf{t}\right)=(-1)^{m} \alpha \delta_{\sigma}\left(-\gamma_{m}\right) \delta(x) E^{+}\left(x, \gamma_{m-2} ; t_{0}, u_{0}, t_{1}+1, u_{1}\right)
$$

where

$$
\alpha=\alpha(\mathbf{t})=\frac{1}{(a+b)(a+b+1)(a+c)(a+d)} .
$$

Here we use the convention $E^{+}\left(x, \gamma_{-1}\right)=1$.

Proof. From Lemma 3.6 and $b_{2 n}-t_{1}=c_{1}^{\sigma}\left(-\gamma_{2 n}\right)$ we find

$$
C_{-} p_{2 n}=-\frac{c_{1}^{\sigma}\left(-\gamma_{2 n}\right)}{2 t_{1}} P_{2 n}^{-}, \quad C_{-} p_{2 n-1}=\frac{1}{2 t_{1}} P_{2 n}^{-}
$$

So by Theorem 3.10 we have $E^{-}\left(x, \gamma_{m}\right)=\alpha_{m} \delta(x) E^{+}\left(x, \gamma_{m-2} ; t_{0}, u_{0}, t_{1}+1, u_{1}\right)$ for $m \in \mathbb{N}$. Recall here that $E^{+}\left(x, \gamma_{2 n}\right)=E^{+}\left(x, \gamma_{2 n-1}\right)$. Normalizing the polynomials then gives

$$
\begin{aligned}
\alpha_{2 n} & =-\frac{c_{1}^{\sigma}\left(-\gamma_{2 n}\right) P_{2 n-2}^{+}\left(x_{0}+1 ; t_{0}, u_{0}, t_{1}+1, u_{1}\right)}{2 t_{1} p_{2 n}\left(-x_{0}\right)}, \\
\alpha_{2 n-1} & =\frac{P_{2 n-2}^{+}\left(x_{0}+1 ; t_{0}, u_{0}, t_{1}+1, u_{1}\right)}{2 t_{1} p_{2 n-1}\left(-x_{0}\right)} .
\end{aligned}
$$

Writing this out explicitly using Proposition 3.14 completes the proof. 
Proposition 5.8. Let $\mathfrak{E}$ be given by

$$
\mathfrak{E}(x, \lambda)=\phi_{\lambda}(x ; \mathbf{t})+\delta(x) \delta_{\sigma}(\lambda) \phi_{\lambda}\left(x ; t_{0}, u_{0}, t_{1}+1, u_{1}\right),
$$

then

$$
\left\langle E_{\tau}(\cdot, \gamma), \mathfrak{E}(\cdot, \lambda)\right\rangle_{\mathbf{t}^{\tau}}=2 t_{1} \frac{G_{\tau \sigma \tau}(\gamma)}{G_{\tau \sigma \tau}\left(\gamma_{0}^{\tau}\right)} E_{\sigma \tau}(\lambda, \gamma), \quad \gamma \in-\Gamma_{\tau} .
$$

Consequently, $(\mathfrak{F} p)(\lambda)=\mathcal{K}\langle p, \mathfrak{E}(\cdot, \lambda)\rangle_{\mathbf{t}^{\tau}}$ for $p \in \mathcal{A}$, where the constant is explicitly given by $\mathcal{K}=\mathcal{N}_{\tau} G_{\tau \sigma \tau}\left(\gamma_{0}^{\tau}\right) / 2 t_{1}$.

Proof. If (5.8) is valid, then by Proposition 5.3(a) we have $(\mathfrak{F} p)(\lambda)=\mathcal{K}\langle p, \mathfrak{E}(\cdot, \lambda)\rangle_{\mathbf{t}^{\tau}}$ for all $p \in \mathcal{A}$, with $\mathcal{K}=\mathcal{N}_{\tau} G_{\tau \sigma \tau}\left(\gamma_{0}^{\tau}\right) / 2 t_{1}$. So we need to show that (5.8) holds.

Let $\gamma \in-\Gamma_{\tau}$. In order to prove (5.8) we split $E_{\tau}$ and $\mathfrak{E}$ in symmetric and antisymmetric parts, then

$$
\left\langle E_{\tau}(\cdot, \gamma), \mathfrak{E}(\cdot, \lambda)\right\rangle_{\mathbf{t}^{\tau}}=\left\langle E_{\tau}^{+}(\cdot, \gamma), \mathfrak{E}^{+}(\cdot, \lambda)\right\rangle_{\mathbf{t}^{\tau}}+\left\langle E_{\tau}^{-}(\cdot, \gamma), \mathfrak{E}^{-}(\cdot, \lambda)\right\rangle_{\mathbf{t}^{\tau}},
$$

where we denoted $C_{ \pm}^{\tau} \mathfrak{E}(\cdot, \lambda)=\mathfrak{E}^{ \pm}(\cdot, \lambda)$. Since $\mathfrak{E}^{+}(x, \lambda)=\phi_{\lambda}(x)$ we obtain, from Lemmas 3.8 and $5.6(\mathrm{c})$,

$$
\left\langle E_{\tau}^{+}(\cdot, \gamma), \mathfrak{E}^{+}(\cdot, \lambda)\right\rangle_{\mathbf{t}^{\tau}}=t_{1}\left\langle E_{\tau}^{+}(\cdot, \gamma), \mathfrak{E}^{+}(\cdot, \lambda)\right\rangle_{\mathbf{t}^{\tau}}^{+}=2 t_{1} \frac{G_{\tau \sigma \tau}(\gamma)}{G_{\tau \sigma \tau}\left(\gamma_{0}\right)} E_{\sigma \tau}^{+}(\lambda, \gamma) .
$$

For $\gamma=\gamma_{0}^{\tau}$ the proposition is now proved, since $E_{\tau}^{-}\left(x, \gamma_{0}^{\tau}\right)=0$. Assume that $\gamma=\gamma_{m}^{\tau}$ for some $m \in \mathbb{N}$. For the antisymmetric part we rewrite $E_{\tau}^{-}$using Lemma 5.7 (note that $\left.\delta=\delta_{\tau}\right)$, and we use $\delta(x) \Delta_{\tau}(x)=-(2 x)^{-1} \Delta^{+}\left(x ; u_{0}, t_{0}, t_{1}+1, u_{1}\right)$, then symmetrizing the integrand gives us

$$
\begin{aligned}
\left\langle E_{\tau}^{-}(\cdot, \gamma), \mathfrak{E}^{-}(\cdot, \lambda)\right\rangle_{\mathbf{t}^{\tau}} & (-1)^{m} \frac{\alpha_{\tau} \delta_{\tau \sigma}(-\gamma) \delta_{\sigma}(\lambda)}{4 \pi i} \int_{\mathcal{C}_{\tau}} E^{+}\left(\cdot, \gamma_{m-2}^{\tau} ; t_{0}, u_{0}, t_{1}+1, u_{1}\right) \phi_{\lambda}\left(x ; t_{0}, u_{0}, t_{1}+1, u_{1}\right) \\
= & \frac{\delta(-x)-\delta(x)}{2 x} \Delta^{+}\left(x ; u_{0}, t_{0}, t_{1}+1, u_{1}\right) d x \\
= & (-1)^{m+1} 2 t_{1} \alpha_{\tau} \delta_{\tau \sigma}(-\gamma) \delta_{\sigma}(\lambda) \\
& \times\left\langle E^{+}\left(\cdot, \gamma_{m-2}^{\tau} ; u_{0}, t_{0}, t_{1}+1, u_{1}\right), \phi_{\lambda}\left(\cdot ; t_{0}, u_{0}, t_{1}+1, u_{1}\right)\right\rangle_{\left(u_{0}, t_{0}, t_{1}+1, u_{1}\right)}^{+} \\
= & (-1)^{m+1} 2 t_{1} \alpha_{\tau} \delta_{\tau \sigma}(-\gamma) \delta_{\sigma}(\lambda) \frac{G_{\tau \sigma \tau}(\gamma)}{G_{\tau \sigma \tau}\left(\gamma_{2}^{\tau}\right)} E^{+}\left(\lambda, \gamma_{m-2}^{\tau} ; u_{0}, u_{1}, t_{1}+1, t_{0}\right) .
\end{aligned}
$$

Here we used $\gamma_{m-2}\left(u_{0}, t_{0}, t_{1}+1, u_{1}\right)= \pm\left(u_{0}+t_{1}+n\right)=\gamma_{m}^{\tau}$ for $m=2 n, 2 n-1$. From the explicit expressions for $\alpha_{\tau}$ and $G_{\tau \sigma \tau}\left(\gamma_{2}^{\tau}\right)$ we find

$$
\frac{\alpha_{\tau}}{G_{\tau \sigma \tau}\left(\gamma_{2}^{\tau}\right)}=-\frac{\alpha_{\sigma \tau}}{G_{\tau \sigma \tau}\left(\gamma_{0}^{\tau}\right)}
$$

Then adding the symmetric and the antisymmetric parts we obtain

$$
\begin{aligned}
\left\langle E_{\tau}(\cdot, \gamma),\right. & \mathfrak{E}(\cdot, \lambda)\rangle_{\mathbf{t}^{\tau}}=2 t_{1} \frac{G_{\tau \sigma \tau}(\gamma)}{G_{\tau \sigma \tau}\left(\gamma_{0}^{\tau}\right)} \\
& \times\left(E_{\sigma \tau}^{+}\left(\lambda, \gamma_{m}^{\tau}\right)+(-1)^{m} \alpha_{\sigma \tau} \delta_{\sigma \tau \sigma}(-\gamma) \delta_{\sigma \tau}(\lambda) E^{+}\left(\lambda, \gamma_{m-2}^{\tau} ; u_{0}, u_{1}, t_{1}+1, t_{0}\right)\right)
\end{aligned}
$$

Note that $\delta_{\tau \sigma}=\delta_{\sigma \tau \sigma}$ and $\delta_{\sigma}=\delta_{\sigma \tau}$, so applying Lemma 5.7 now gives (5.8). 
Now we can express $\mathfrak{F}$, and therefore also $\mathcal{F}^{\prime}$, as an integral transform. Our next goal is to write $\mathcal{F}^{\prime}$ in the form $\left(\mathcal{F}^{\prime} f\right)(\lambda)=\{p, \mathcal{E}(\cdot, \lambda)\}$, where the bilinear form $\{\cdot, \cdot\}$ is such that the generators of $\mathcal{H}$ are symmetric with respect to this bilinear form. The intertwining property $\mathcal{F}^{\prime} \circ X=\sigma(X) \circ \mathcal{F}^{\prime}, X \in \mathcal{H}$, then immediately gives transformation properties of the kernel $\mathcal{E}$ under the action of $\mathcal{H}$ and $\mathcal{H}_{\sigma}$.

Let $\Theta$ be the weight function given by

$$
\Theta(x)=G_{\tau}(x)^{-1} G(x)^{-1} \Delta(x) .
$$

By Euler's reflection formula for the $\Gamma$-function we may write

$$
G_{\tau}(x)^{-1} G(x)^{-1}=\frac{1}{\pi^{2}} \sin \pi(d \pm x),
$$

and from this expression it is easy to see that $G_{\tau}^{-1} G^{-1}$ is $\mathcal{W}$-invariant. To the weight function $\Theta$ we associate a bilinear form $\{\cdot, \cdot\}$ on $\mathcal{A} G_{\tau}$;

$$
\{f, g\}_{\mathbf{t}}=\frac{1}{2 \pi i} \int_{\mathcal{C}} f(x) g(x) \Theta(x) d x .
$$

Lemma 5.9. The algebraic generators $z, T_{0}, T_{1}$ of $\mathcal{H}$ are symmetric with respect to $\{\cdot, \cdot\}_{\mathbf{t}}$.

Proof. For $z$ this is trivial. For $i=0,1$, we have

$$
T_{i}\left(G_{\tau}(x)^{-1} G(x)^{-1} f\right)=G_{\tau}(x)^{-1} G(x)^{-1} T_{i} f,
$$

since $G_{\tau}(x)^{-1} G(x)^{-1}$ is $\mathcal{W}$-invariant. Since $T_{i}$ is symmetric with respect $\langle\cdot, \cdot\rangle_{\mathbf{t}}$ we have $\left\{T_{i} f, g\right\}_{\mathbf{t}}=\left\langle T_{i} f, G_{\tau}^{-1} G^{-1} g\right\rangle_{\mathbf{t}}=\left\langle f, T_{i}\left(G_{\tau}^{-1} G^{-1} g\right)\right\rangle_{\mathbf{t}}=\left\langle f, G_{\tau}^{-1} G^{-1} T_{i} g\right\rangle_{\mathbf{t}}=\left\{f, T_{i} g\right\}_{\mathbf{t}}$.

With the bilinear form $\{\cdot, \cdot\}$ we are going to construct a Fourier transform. The following function is the kernel in this Fourier transform.

Definition 5.10. We define the (nonsymmetric) Wilson function by

$$
\mathcal{E}(x, \lambda)=G_{\tau}(x) G_{\sigma \tau}(\lambda) \mathfrak{E}(x, \lambda) .
$$

Observe that $\mathfrak{E}(x, \lambda)$ is an entire function in $x$ and $\lambda$, so the Wilson function is a meromorphic function in $x$ and $\lambda$ with simple poles coming from the Gaussians, located at $x= \pm(1-d+n), \lambda= \pm(1-\tilde{d}+n), n \in \mathbb{Z}_{\geqslant 0}$. With the nonsymmetric Wilson function we now define an integral transform that is a Fourier transform associated to the duality isomorphism $\sigma$. Moreover, we can find the inverse and Plancherel-type formulas.

Theorem 5.11. Let $\mathcal{F}$ be the linear operator defined by

$$
(\mathcal{F} f)(\lambda)=\{f, \mathcal{E}(\cdot, \lambda)\}_{\mathbf{t}}, \quad f \in \mathcal{A} G_{\tau} .
$$

Then:

(a) $\mathcal{F}$ maps $\mathcal{A} G_{\tau}$ into $\mathcal{A} G_{\sigma \tau}$.

(b) $\mathcal{F}$ is a Fourier transform associated to $\sigma$.

(c) $\mathcal{F} \circ \mathcal{F}_{\sigma}=(a+b)^{2} i d_{\mathcal{A} G_{\sigma \tau}}$ and $\mathcal{F}_{\sigma} \circ \mathcal{F}=(a+b)^{2} i d_{\mathcal{A} G_{\tau}}$.

(d) For $f_{1}, f_{2} \in \mathcal{A} G_{\tau}$ and $g_{1}, g_{2} \in \mathcal{A} G_{\sigma \tau}$,

$$
\left\{\mathcal{F} f_{1}, \mathcal{F} f_{2}\right\}_{\mathbf{t}^{\sigma}}=(a+b)^{2}\left\{f_{1}, f_{2}\right\}_{\mathbf{t}}, \quad\left\{\mathcal{F}_{\sigma} g_{1}, \mathcal{F}_{\sigma} g_{2}\right\}_{\mathbf{t}}=(a+b)^{2}\left\{g_{1}, g_{2}\right\}_{\mathbf{t}^{\sigma}} .
$$


Proof. (a) We only need to check that the operator $\mathcal{F}$ is the same as $k \mathcal{F}^{\prime}$, see (5.3), for some nonzero constant $k$. Let $f \in \mathcal{A} G_{\tau}$. Observe that

$$
\Delta_{\tau}(x)=G_{\tau}(x) G(x)^{-1} \Delta(x),
$$

then using Proposition 5.8 we have

$$
\begin{aligned}
\mathcal{K}^{-1}\left(G_{\sigma \tau} \circ \mathfrak{F} \circ G_{\tau}^{-1}\right) f(\lambda) & =G_{\sigma \tau}(\lambda)\left\langle G_{\tau}^{-1} f, \mathfrak{E}(\cdot, \lambda)\right\rangle_{\mathbf{t}^{\tau}} \\
& =G_{\sigma \tau}(\lambda)\left\langle G_{\tau}^{-1} G^{-1} f, G_{\tau} \mathfrak{E}(\cdot, \lambda)\right\rangle_{\mathbf{t}} \\
& =\{f, \mathcal{E}(\cdot, \lambda)\}_{\mathbf{t}}
\end{aligned}
$$

This is the desired result.

(b) This follows from the proof of (a) and Proposition 5.4.

(c) Let us denote $e_{\gamma}(x)=G_{\tau}(x) E_{\tau}(x, \gamma)$ for $\gamma \in-\Gamma_{\tau}$. From Proposition 5.8 we obtain

$$
\left(\mathcal{F} e_{\gamma}\right)(\lambda)=(a+b) \frac{G_{\tau \sigma \tau}(\gamma)}{G_{\tau \sigma \tau}\left(\gamma_{0}^{\tau}\right)} G_{\sigma \tau}(\lambda) E_{\sigma \tau}(\lambda, \gamma)=(a+b) \frac{G_{\tau \sigma \tau}(\gamma)}{G_{\tau \sigma \tau}\left(\gamma_{0}^{\tau}\right)} e_{\gamma}^{\sigma}(\lambda)
$$

Next we apply $\mathcal{F}_{\sigma}: \mathcal{A} G_{\sigma \tau} \rightarrow \mathcal{A} G_{\tau}$, which comes down to replacing $\mathbf{t}$ by $\mathbf{t}^{\sigma}$, then

$$
\begin{aligned}
\left(\mathcal{F}_{\sigma} \circ \mathcal{F}\right)\left(e_{\gamma}\right)(x) & =(a+b) \frac{G_{\tau \sigma \tau}(\gamma)}{G_{\tau \sigma \tau}\left(\gamma_{0}^{\tau}\right)}\left(\mathcal{F}_{\sigma} e_{\gamma}^{\sigma}\right)(x) \\
& =(a+b)^{2} \frac{G_{\tau \sigma \tau}(\gamma) G_{\tau \sigma}(\gamma)}{G_{\tau \sigma \tau}\left(\gamma_{0}^{\tau}\right) G_{\tau \sigma}\left(\gamma_{0}^{\tau}\right)} e_{\gamma}(x),
\end{aligned}
$$

where we used $\mathbf{t}^{\sigma \tau \sigma \tau}=\mathbf{t}^{\tau \sigma}$. Writing out the Gaussians explicitly we have, for $m=2 n$ or $m=2 n-1$,

$$
\frac{G_{\tau \sigma}\left(\gamma_{m}^{\tau}\right)}{G_{\tau \sigma}\left(\gamma_{0}^{\tau}\right)}=(-1)^{n} \frac{(a+1-d)_{n}}{(b+c)_{n}}, \quad \frac{G_{\tau \sigma \tau}\left(\gamma_{m}^{\tau}\right)}{G_{\tau \sigma \tau}\left(\gamma_{0}^{\tau}\right)}=(-1)^{n} \frac{(b+c)_{n}}{(a+1-d)_{n}}
$$

so we have $\left(\mathcal{F}_{\sigma} \circ \mathcal{F}\right)\left(e_{\gamma}\right)(x)=(a+b)^{2} e_{\gamma}(x)$. Since the nonsymmetric Wilson polynomials form a basis for $\mathcal{A}$, the set $\left\{e_{\gamma} \mid \gamma \in-\Gamma_{\tau}\right\}$ is a basis for $\mathcal{A} G_{\tau}$, and therefore $\mathcal{F}_{\sigma} \circ \mathcal{F}=$ $(a+b)^{2} i d_{\mathcal{A} G_{\tau}}$. In the same way we obtain $\mathcal{F} \circ \mathcal{F}_{\sigma}=(a+b)^{2} i d_{\mathcal{A} G_{\sigma \tau}}$.

(d) It is enough to show that the Plancherel formula is valid for the basis elements $e_{\gamma}$ of $\mathcal{A} G_{\tau}$. By Theorem 4.3 we have, for $\gamma, \gamma^{\prime} \in-\Gamma_{\tau}$,

$$
\left\{e_{\gamma}, e_{\gamma^{\prime}}\right\}_{\mathbf{t}}=\left\langle E_{\tau}(\cdot, \gamma), E_{\tau}\left(\cdot, \gamma^{\prime}\right)\right\rangle_{\mathbf{t}^{\tau}}=\delta_{\gamma, \gamma^{\prime}} \frac{\langle 1,1\rangle_{\mathbf{t}^{\tau}} w_{\tau}\left(-\gamma_{0}^{\tau}\right)}{w_{\tau}(-\gamma)}
$$

Writing out explicitly $\langle 1,1\rangle_{\mathbf{t}^{\tau}}$ in terms of $t_{0}, u_{0}, t_{1}, u_{1}$ using (4.2) and (3.1), we find an expression that is invariant under $t_{0} \leftrightarrow u_{1}$, so

$$
\langle 1,1\rangle_{\mathbf{t}^{\tau}}=\langle 1,1\rangle_{\mathbf{t}^{\sigma \tau}}
$$


Using also the identity $G_{\tau \sigma \tau}(\gamma) w_{\tau}(\gamma)=G_{\tau \sigma}(\gamma) w_{\sigma \tau}(\gamma)$, see (5.2), we then obtain

$$
\begin{aligned}
\left\{e_{\gamma}, e_{\gamma^{\prime}}\right\}_{\mathbf{t}} & =\delta_{\gamma, \gamma^{\prime}} \frac{k_{1}(\gamma)\langle 1,1\rangle_{\mathbf{t}^{\sigma \tau}} w_{\sigma \tau}\left(-\gamma_{0}^{\tau}\right)}{w_{\sigma \tau}(-\gamma)} \\
& =k_{1}(\gamma)\left\langle E_{\sigma \tau}(\cdot, \gamma), E_{\sigma \tau}\left(\cdot, \gamma^{\prime}\right)\right\rangle_{\mathbf{t}^{\sigma \tau}}=k_{1}(\gamma)\left\{e_{\gamma}^{\sigma}, e_{\gamma^{\prime}}^{\sigma}\right\}_{\mathbf{t}^{\sigma}},
\end{aligned}
$$

where

$$
k_{1}(\gamma)=\frac{G_{\tau \sigma}\left(\gamma_{0}^{\tau}\right) G_{\tau \sigma \tau}(\gamma)}{G_{\tau \sigma}(\gamma) G_{\tau \sigma \tau}\left(\gamma_{0}^{\tau}\right)}
$$

Now from (5.9) it follows that

$$
\left\{e_{\gamma}, e_{\gamma^{\prime}}\right\}_{\mathbf{t}}=k_{2}(\gamma)\left\{\mathcal{F} e_{\gamma}, \mathcal{F} e_{\gamma^{\prime}}\right\}_{\mathbf{t}^{\sigma}},
$$

where the factor $k_{2}(\gamma)$ is given by

$$
k_{2}(\gamma)=\frac{k_{1}(\gamma) G_{\tau \sigma \tau}\left(\gamma_{0}^{\tau}\right)^{2}}{(a+b)^{2} G_{\tau \sigma} \tau(\gamma)^{2}}=(a+b)^{-2} \frac{G_{\tau \sigma}\left(\gamma_{0}^{\tau}\right) G_{\tau \sigma \tau}\left(\gamma_{0}^{\tau}\right)}{G_{\tau \sigma}(\gamma) G_{\tau \sigma \tau}(\gamma)}
$$

By (5.10) we have $k_{2}(\gamma)=(a+b)^{-2}$, so we have proved the first Plancherel formula. The second formula follows from applying (c).

Let us define the symmetric Wilson function by

$$
\mathcal{E}^{+}(x, \lambda)=\left(C_{+} \mathcal{E}(\cdot, \lambda)\right)(x) .
$$

Observe that $\mathcal{E}^{+}(x, \lambda)=G_{\tau}(x) G_{\sigma \tau}(\lambda) \phi_{\lambda}(x)$, with $\phi_{\lambda}$ defined by (5.6). So $\phi_{\lambda}$ can be considered as the analytic part of the symmetric Wilson function. With the symmetric kernel $\mathcal{E}^{+}$we define the integral transform

$$
\left(\mathcal{F}^{+} f\right)(\lambda)=\left\{f, \mathcal{E}^{+}(\cdot, \lambda)\right\}_{\mathbf{t}}^{+}, \quad f \in \mathcal{A}_{+} G_{\tau},
$$

where $\{\cdot, \cdot\}_{\mathbf{t}}^{+}$is the bilinear form on $\mathcal{A}_{+} G_{\tau}$ defined by

$$
\begin{aligned}
\{f, g\}_{\mathbf{t}}^{+} & =\frac{1}{4 \pi i} \int_{\mathcal{C}} f(x) g(x) \Theta^{+}(x) d x \\
\Theta^{+}(x) & =G_{\tau}(x)^{-1} G(x)^{-1} \Delta^{+}(x)
\end{aligned}
$$

Then it not hard to verify that $\mathcal{F}^{+}: \mathcal{A}_{+} G_{\tau} \rightarrow \mathcal{A}_{+} G_{\sigma \tau}$ has the inverse $\mathcal{F}_{\sigma}^{+}$. Moreover, we have the Plancherel-type formula

$$
\left\{\mathcal{F}^{+} f_{1}, \mathcal{F}^{+} f_{2}\right\}_{\mathbf{t}^{\sigma}}^{+}=\left\{f_{1}, f_{2}\right\}_{\mathbf{t}}^{+}, \quad f_{1}, f_{2} \in \mathcal{A}_{+} G_{\tau}
$$

so $\mathcal{F}^{+}$is an isometry.

Let us assume that the parameters $a, b, c, 1-d$ are positive, or the nonreal parameters occur in pairs of complex conjugates with positive real parts. Now the weights $\Theta^{+}$and $\Delta_{\tau}^{+}$are positive on $i \mathbb{R}$, so $\{\cdot, \cdot\}_{\mathbf{t}}^{+}$and $\langle\cdot, \cdot\rangle_{\mathbf{t}^{\tau}}^{+}$define inner products. In general, if for some $\varepsilon>0$ we have

$$
\int_{\mathbb{R}} e^{\varepsilon|x|} d \mu(x)<\infty
$$


then the moment problem for the measure $\mu$ is determinate, see [10] and references therein, and therefore the polynomials are dense in the Hilbert space $L^{2}(\mathbb{R}, \mu)$. Using the asymptotic behavior (5.6) of $\Delta_{\tau}^{+}$we find, for $0<\varepsilon<2 \pi$,

$$
\int_{\mathbb{R}} e^{\varepsilon|x|} \Delta_{\tau}^{+}(i x) d x<\infty
$$

so the polynomials are dense in $L^{2}\left(i \mathbb{R}, \Delta_{\tau}^{+}\right)$. In particular, the symmetric Wilson polynomials $E_{\tau}^{+}(x, \gamma), \gamma \in \Gamma^{+}$, form an orthogonal basis for $L^{2}\left(i \mathbb{R}, \Delta_{\tau}^{+}\right)^{W}$, the subspace of even functions in $L^{2}\left(i \mathbb{R}, \Delta_{\tau}^{+}\right)$. From this it follows that the set $\mathcal{A}_{+} G_{\tau}$ is dense in $L^{2}\left(i \mathbb{R}, \Theta^{+}\right)^{W}$. Therefore the integral transform $\mathcal{F}^{+}$extends uniquely to a unitary operator $\tilde{\mathcal{F}}^{+}: L^{2}\left(i \mathbb{R},(4 \pi i)^{-1} \Theta^{+}\right)^{W} \rightarrow L^{2}\left(i \mathbb{R},(4 \pi i)^{-1} \Theta_{\sigma}^{+}\right)^{W}$. Also, the operator $\mathfrak{F}^{+}=G_{\sigma \tau}^{-1} \circ \mathcal{F}^{+} \circ G_{\tau}$ extends uniquely to a unitary operator $\tilde{\mathfrak{F}}^{+}: L^{2}\left(i \mathbb{R},(4 \pi i)^{-1} \Delta_{\tau}^{+}\right)^{W} \rightarrow$ $L^{2}\left(i \mathbb{R},(4 \pi i)^{-1} \Delta_{\sigma \tau}^{+}\right)^{W}$. This operator $\tilde{\mathfrak{F}}^{+}$is precisely the Wilson function transform of type I, as defined in [8].

\subsection{Properties of the Wilson function}

Let us finish this paper with a few nice properties of the Wilson function. From the property $\mathcal{F} \circ X=\sigma(X) \circ \mathcal{F}$ and Lemma 5.9 it follows immediately that the nonsymmetric Wilson function satisfies

$$
(X \mathcal{E}(\cdot, \lambda))(x)=(\psi(X) \mathcal{E}(x, \cdot))(\lambda), \quad X \in \mathcal{H}
$$

where $\psi$ denotes the duality anti-isomorphism. In particular, $\mathcal{E}(x, \lambda)$ is an eigenfunction of the Dunkl-Cherednik-type operators $Y$ and $Y^{\sigma}$ for eigenvalue $-\lambda$, respectively, $-x$. From (the proof of) Proposition 4.1 it follows that for $\lambda=\gamma \in \Gamma$ the Wilson polynomial $E(x,-\gamma)$ also satisfies the transformation property $(5.11)$. This suggests that $\mathcal{E}(x, \gamma)=$ $k E(x,-\gamma), \gamma \in \Gamma$, for some nonzero constant $k$. Moreover, the dual Wilson function $\mathcal{E}_{\sigma}(\lambda, x)$ also satisfies $(5.11)$, which suggests that $\mathcal{E}(x, \lambda)=k \mathcal{E}_{\sigma}(\lambda, x)$ for some nonzero constant $k$.

Theorem 5.12. The Wilson function $\mathcal{E}(x, \lambda)$ satisfies:

(a) $\mathcal{E}(x, \gamma)=\frac{\Gamma(1-a-d)}{\Gamma(a+b) \Gamma(a+c)} E(x,-\gamma)$ for $\gamma \in \Gamma$;

(b) $\mathcal{E}(x, \lambda)=\mathcal{E}_{\sigma}(\lambda, x)$.

So $\mathcal{E}(x, \lambda)$ is a meromorphic continuation of the nonsymmetric Wilson polynomial in its degree, and like the Wilson polynomial, $\mathcal{E}(x, \lambda)$ also satisfies a duality property.

Proof. Consider first the function $\phi_{\lambda}(x)$ defined by (5.5). Using $a+e=\tilde{a}+\tilde{e}$ for $e=b, c, d$ and $e-d=\tilde{e}-\tilde{d}$ for $e=b, c$, the duality property for $\phi_{\lambda}$ follows directly from $(5.5)$ :

$$
\phi_{\lambda}(x ; \mathbf{t})=\phi_{x}\left(\lambda ; \mathbf{t}^{\sigma}\right) .
$$

Now the duality property for the Wilson function follows from Definition 5.10 and the definition of the kernel $\mathfrak{E}$ in Proposition 5.8. 
For the reduction to the Wilson polynomials observe the function $\Gamma(\tilde{a} \pm \lambda)^{-1}$ has zeros at $\lambda= \pm(\tilde{a}+n), n \in \mathbb{Z}_{\geqslant 0}$. Thefore the second ${ }_{4} F_{3}$-function in (5.5) vanishes for $\lambda \in \Gamma$, and then we see that

$$
\phi_{\gamma}(x)=\frac{\Gamma(1-a-d)}{\Gamma(a+b) \Gamma(a+c) G_{\tau}(x) G_{\sigma \tau}(\gamma)} E^{+}(x, \gamma), \quad \gamma \in \Gamma .
$$

Now (a) follows from Definition 5.10 and Lemma 5.7.

\section{References}

[1] R. Askey, J. Wilson, Some Basic Hypergeometric Orthogonal Polynomials That Generalize Jacobi Polynomials, Mem. Amer. Math. Soc. 54 (1985).

[2] I. Cherednik, Nonsymmetric Macdonald polynomials, Int. Math. Res. Not. 10 (1995), 483515.

[3] I. Cherednik, Difference Macdonald-Mehta conjecture, Int. Math. Res. Not. 10 (1997), 449-467.

[4] I. Cherednik, Inverse Harish-Chandra transform and difference operators, Int. Math. Res. Not. 15 (1997), 733-750.

[5] I. Cherednik, Y. Markov, Hankel transform via double Hecke algebra, in: Iwahori-Hecke Algebras and Their Representation Theory (Martina-Franca, 1999), pp. 1-25, Lecture Notes in Mathematics, Vol. 1804, Springer-Verlag, New York, 2002.

[6] W. Crawley-Boevey, M. P. Holland, Noncommutative deformations of Kleinian singularities, Duke Math. J. 92 (1998), 605-635.

[7] P. Etingof, A. Oblomkov, E. Rains, Generalized double affine Hecke algebras of rank 1 and quantized Del Pezzo surfaces, math.QA/0406480.

[8] W. Groenevelt, The Wilson function transform, Int. Math. Res. Not. 52 (2003), 27792817.

[9] M. E. H. Ismail, J. Letessier, G. Valent, J. Wimp, Two families of associated Wilson polynomials, Canad. J. Math. 42 (1990), 659-695.

[10] M. de Jeu, Determinate multidimensional measures, the extended Carleman theorem and quasi-analytic weights, Ann. Probab. 31 (2003), 1205-1227.

[11] E. Koelink, J. V. Stokman, The Askey-Wilson function transform, Int. Math. Res. Not. 22 (2001), 1203-1227.

[12] T. H. Koornwinder, Special orthogonal polynomial systems mapped onto each other by the Fourier-Jacobi transform, in: Orthogonal Polynomials and Applications (Bar-le-Duc, 1984), pp. 174-183, Lecture Notes in Mathematics, Vol. 1171, Springer-Verlag, New York, 1985.

[13] T. H. Koornwinder, Askey-Wilson polynomials for root systems of type BC, Contemp. Math. 138 (1992), 189-204.

[14] D. R. Masson, Associated Wilson polynomials, Constr. Approx. 7 (1991), 521-534.

[15] I. G. Macdonald, Affine Hecke Algebras and Orthogonal Polynomials, Cambridge Tracts in Mathematics, Vol. 157, Cambridge University Press, Cambridge, 2003.

[16] A. Mellit, Algebras generated by elements with given spectrum and scalar sum and Kleinian singularities, math.RA/0406119. 
[17] M. Noumi, Macdonald-Koornwinder polynomials and affine Hecke rings, Sūrikaisekikenkyūsho Kōkyūroku 919 (1995), 44-55 (in Japanese).

[18] M. Noumi, J. V. Stokman, Askey-Wilson polynomials: An affine Hecke algebra approach, in: Laredo Lectures on Orthogonal Polynomials and Special Functions, pp.111-144, Adv. Theory Spec. Funct. Orthogonal Polynomials, Nova Science, Hauppauge, NY, 2004.

[19] E. M. Opdam, Harmonic analysis for certain representations of graded Hecke algebras, Acta Math. 175 (1995), 75-121.

[20] S. Sahi, Nonsymmetric Koornwinder polynomials and duality, Ann. of Math. 150 (1999), 267-282.

[21] S. Sahi, Some properties of Koornwinder polynomials, in: q-Series from a Contemporary Perspective (South Hadley, MA, 1998), pp.395-411, Contemporary Mathematics, Vol. 254, Amer. Math. Soc., Providence, RI, 2000.

[22] J. V. Stokman, Koornwinder polynomials and affine Hecke algebras, Int. Math. Res. Not. 19 (2000), 1005-1042.

[23] J. V. Stokman, Difference Fourier transforms for nonreduced root systems, Selecta Math. (N.S.) 9 (2003), 409-494.

[24] J. A. Wilson, Some hypergeometric orthogonal polynomials, SIAM J. Math. Anal. 11 (1980), 690-701.

[25] G. Zhang, Spherical transform and Jacobi polynomials on root systems of type BC, Int. Math. Res. Not. 51 (2005), 3169-3189. 